\title{
Development of 3D Anti-Swing Control for Hydraulic Knuckle Boom Crane
}

\author{
K. J. Jensen ${ }^{1}$ M. K. Ebbesen ${ }^{1}$ M. R. Hansen ${ }^{1}$ \\ ${ }^{1}$ Department of Engineering Sciences, University of Agder, 4879 Grimstad, Norway. \\ E-mail: \{konrad.j.jensen, morten.k.ebbesen, michael.r.hansen\} @uia.no
}

\begin{abstract}
In this paper, 3D anti-swing control for a hydraulic loader crane is presented. The difference between hydraulic and electric cranes are discussed to show the challenges associated with hydraulic actuation. The hanging load dynamics and relevant kinematics of the crane are derived to model the system and create the $3 \mathrm{D}$ anti-swing controller. The anti-swing controller generates a set of tool point velocities which are added to the electro-hydraulic motion controller via feedforward. A dynamic simulation model of the crane is made, and the control system is evaluated in simulations with a path controller in actuator space. Simulation results show significant reduction in the load swing angles during motion using the proposed anti-swing controller in addition to pressure feedback. Experiments are carried out to verify the performance of the anti-swing controller. Results show that the implemented pressure feedback is crucial for reaching stability, and with it the control system yields good suppression of the swing angles in practice.
\end{abstract}

Keywords: Loader crane; Anti-swing; Hydraulics; Kinematics; Feedforward; Pressure feedback

\section{Introduction}

Anti-swing control of cranes and hoists is a topic which has received extensive research in the past decades. Applications include lifting equipment in factories, shipyards, and warehouses etc. One of the challenges associated with these machines is the undesirable load swing of the hanging load when moving. The load swing can reduce efficiency, increase cycle times, and potentially lead to safety hazards and accidents. Different techniques have been implemented to dampen this load swing. Anti-swing control is a difficult task, as systems with hanging loads are underactuated, meaning the degrees of freedom are greater than the number of controlled actuators.

A typical application of anti-swing control is electric overhead cranes, where the translational motion is controlled by one or more electric servomotors. The controller often consists of two parts, one feedback con- troller for regulating the position of the crane, and a second controller to suppress the load swing of the hanging load. Earlier work on anti-swing control of electric overhead cranes include Lee et al. (1997); Lee (1998); Sung-Kun Cho and Ho-Hoon Lee (2000); HoHoon Lee and Seung-Gap Choi (2001), utilizing linear system models. More advanced and nonlinear antiswing control systems including sliding mode control, robust control, and fuzzy logic are found in Ho-Hoon Lee and Sung-Kun Cho (2001); Cho and Lee (2002); Lee (2003); Fang et al. (2003); Lee (2004); Lee et al. (2006); Park et al. (2007); Park et al. (2008); Schindele et al. (2009); Lee and Liang (2010); Ngo and Hong (2012); Ambrosino et al. (2020).

A method which has been tested for anti-swing and vibration reduction in flexible systems is called input shaping. The input signal to the system is designed to be self-canceling, using the bandwidth and damping ratio of a dynamic system model Singhose et al. (1996); 
Sorensen et al. (2007); Kjelland and Hansen (2015).

Delayed reference control is a similar method which has been used on cranes. In this case the reference generator is time shifted based on the measured payload angle, see Boschetti et al. (2011, 2014).

Anti-swing control for hydraulic cranes is not an extensively studied area, but references include Kjelland et al. (2012) which investigates tool-point control and anti-swing for a planar hydraulic crane.

For this paper, a hydraulic loader crane is considered, see Figure 2. In this case the load is hanging from the crane tip, instead of the trolley of an overhead crane. The hydraulic loader crane uses pressure compensated valves, which give a load independent velocity control for each actuator. For closed loop control systems, the load independent velocity control can be utilized in a control system using feedforward Bak and Hansen (2013); Jensen et al. (2020a). In this case, both a position reference and a velocity reference are generated in the control system. An example of a typical closed loop electro-hydraulic motion control system with feedforward (FF) and feedback $(\mathrm{FB})$ is shown in Figure 1.

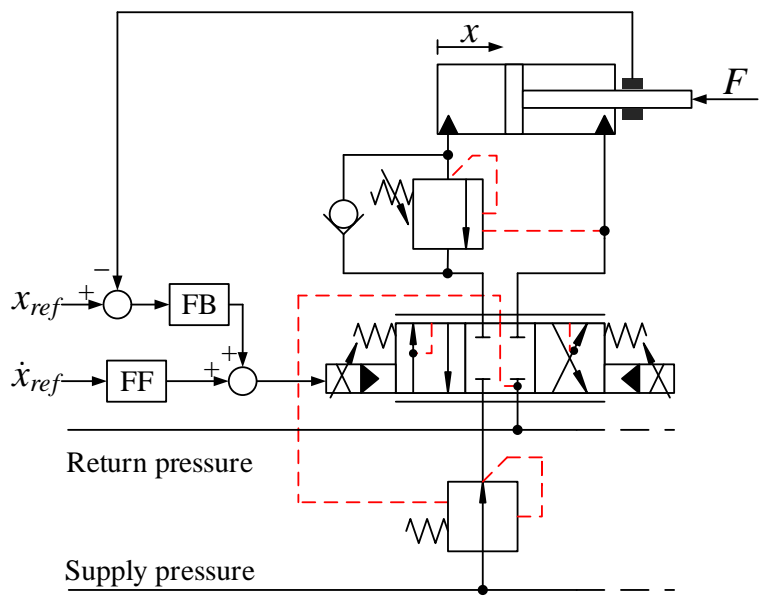

Figure 1: Electro-hydraulic motion control system with feedforward.

This paper is based on the previous work in Jensen et al. (2021), which focus on 2D anti-swing, and extends the relevant kinematics and dynamics in order to create a $3 \mathrm{D}$ anti-swing control system.

\section{Considered System}

In this paper an HMF 2020K4 loader crane is used as a case study for modeling, simulation, and experiments. Figure 2 shows the main components of the HMF 2020K4 loader crane. The relevant data for the cylinders are shown in Table 1. Each actuator is controlled via a pressure compensated proportional directional valve which ensures load independent flow control of the actuators. Counterbalance valves are also used for load holding, assisting in lowering of the booms, and pressure relief of pressure surges. An illustration of the hydraulic system for the knuckle cylinder is shown in Figure 3. Figure 4 shows the hanging load definitions along with the main boom angle $\theta_{m}$, knuckle boom angle $\theta_{k}$, and swing angles $\alpha$ (in-plane angle) and $\beta$ (out-of-plane angle).

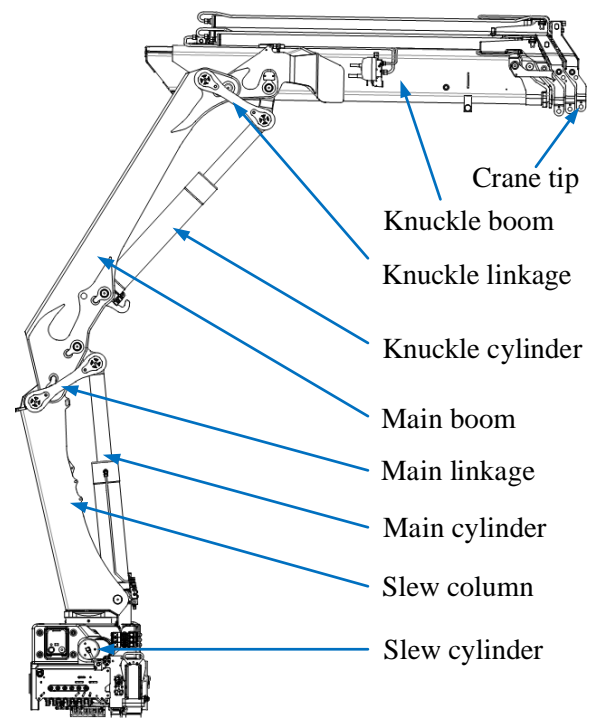

Figure 2: Main components of the considered crane.

Table 1: Data for the cylinders.

\begin{tabular}{lll}
\hline Description & Name & Value \\
\hline Slew piston diameter & $D_{s}$ & $0.125 \mathrm{~m}$ \\
Slew piston area & $A_{s}$ & $0.0123 \mathrm{~m}^{2}$ \\
Slew piston area ratio & $\phi_{s}$ & 1 \\
Slew rack and pinion radius & $r_{s}$ & $0.105 \mathrm{~m}$ \\
Slew valve maximum flow & $Q_{\max , s}$ & $25 \mathrm{l} / \mathrm{min}$ \\
Main piston diameter & $D_{p, m}$ & $0.16 \mathrm{~m}$ \\
Main piston area & $A_{m}$ & $0.0201 \mathrm{~m}^{2}$ \\
Main rod diameter & $D_{r, m}$ & $0.1 \mathrm{~m}$ \\
Main annulus area & $A_{a, m}$ & $0.0123 \mathrm{~m}^{2}$ \\
Main piston area ratio & $\phi_{m}=\frac{A_{a, m}}{A_{m}}$ & 0.6094 \\
Main valve maximum flow & $Q_{\max , m}$ & $40 \mathrm{l} / \mathrm{min}$ \\
Knuckle piston diameter & $D_{p, k}$ & $0.15 \mathrm{~m}$ \\
Knuckle piston area & $A_{k}$ & $0.0177 \mathrm{~m}^{2}$ \\
Knuckle rod diameter & $D_{r, k}$ & $0.1 \mathrm{~m}$ \\
Knuckle annulus area & $A_{a, k}$ & $0.0098 \mathrm{~m}^{2}$ \\
Knuckle piston area ratio & $\phi_{k}=\frac{A_{a, k}}{A_{k}}$ & 0.5556 \\
Knuckle valve maximum flow & $Q_{\max , k}$ & $401 / \mathrm{min}^{2}$ \\
\hline
\end{tabular}




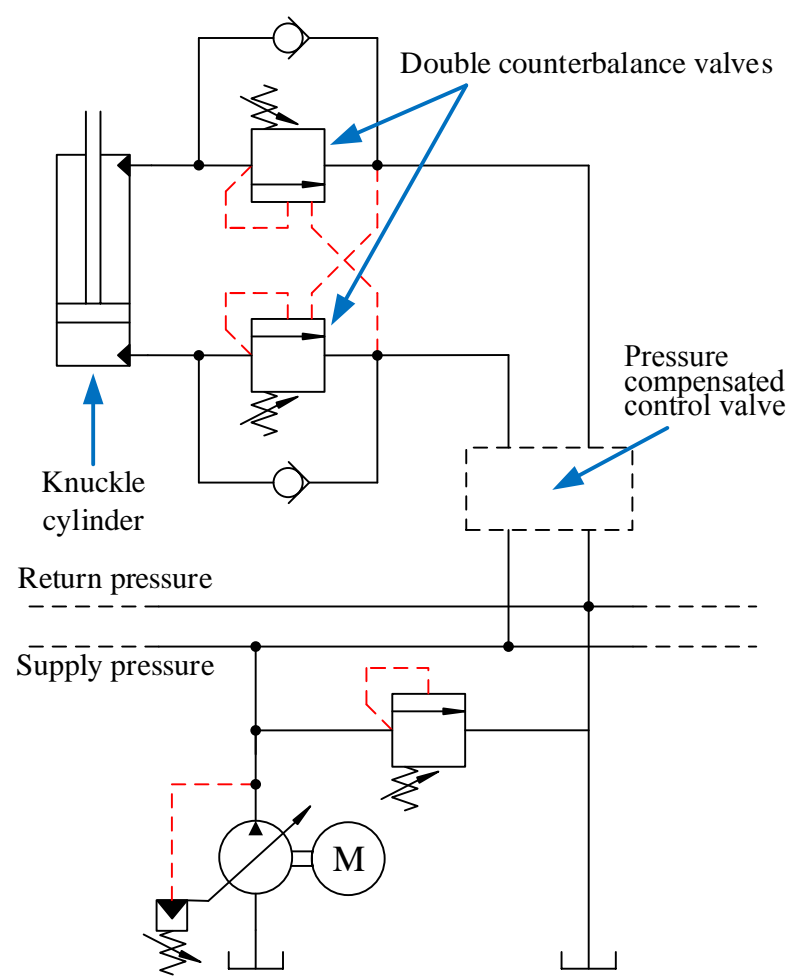

Figure 3: Hydraulic circuit for the knuckle cylinder.

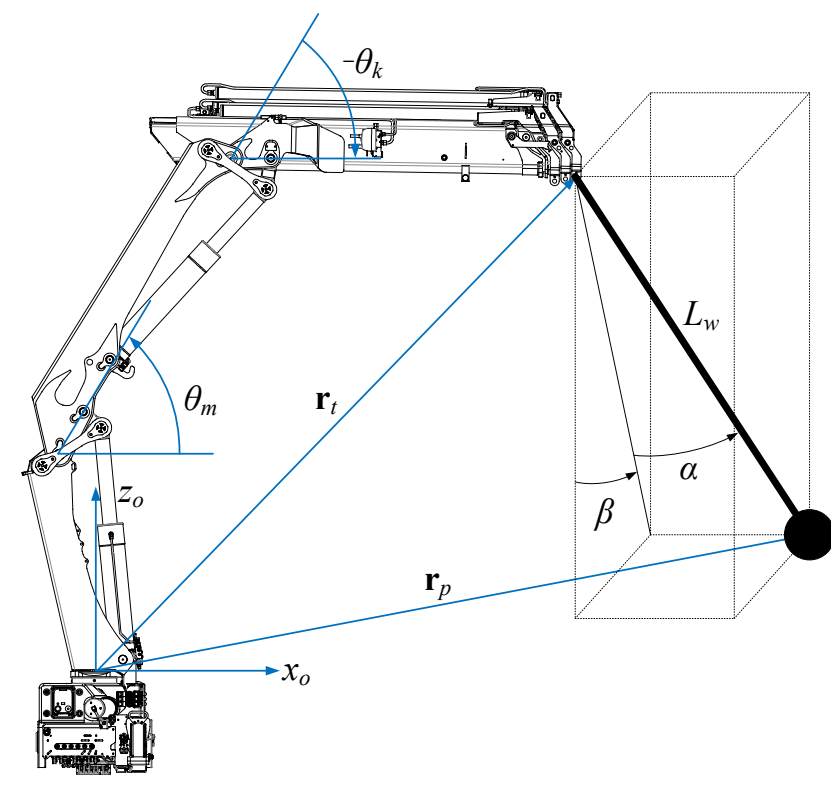

Figure 4: Definitions of crane tip and hanging load geometries.

\subsection{Difference from electric overhead cranes}

Since anti-swing control, primarily of electric overhead cranes, is an extensively studied subject, a short discussion about the differences between electric and hydraulic cranes is presented in this section.

Regarding actuation, the electric motor produces a torque based on the motor current, while hydraulic cylinders produce a force based on the hydraulic pressure. Pressure compensated directional valves are often used on hydraulic cranes, which enable load independent velocity control of the cylinder. On electric cranes, the motor current is controlled to provide a force acting on the system through a rack and pinion. This difference in velocity control and force control also affects the anti-swing control system for the crane.

A typical overhead crane is illustrated in Figure 5. The hanging load is connected to the trolley, and the trolley is able to move in the $x$-direction. An electric motor exerts a force $F_{\text {motor }}$ on the trolley through the wheels, which affects both the trolley motion and payload motion.

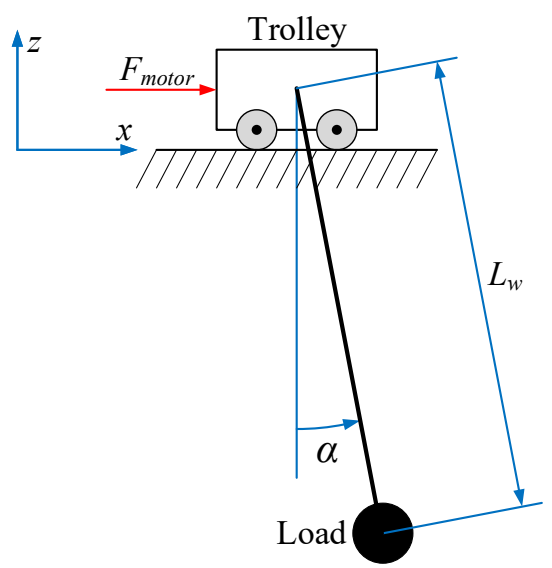

Figure 5: Typical overhead crane with hanging load.

\subsection{Control strategy}

The control strategy suggested in this paper is shown in Figure 6. This control strategy is useful for any hydraulically actuated manipulator with a tool point and a number of joint angles controlled by means of hydraulic cylinders. This constitutes a wide variety of load handling machinery. The main task is position control of the tool point and, classically, this may be combined with a velocity feedforward term. Feedback control is most easily implemented in actuator space Jensen et al. (2020b). Therefore, inverse kinematics is used to transform from tool point coordinates via joint coordinates to actuator coordinates. The anti-swing 
controller is introduced in parallel with the feedforward term, and is used to counteract the continuously measured swing angles by adjusting the tool point velocities. Therefore, the anti-swing controller also requires a kinematic transformation into actuator space.

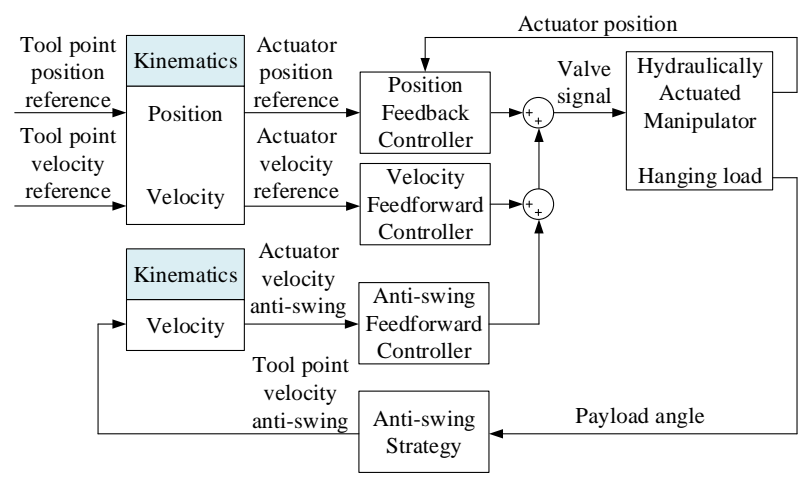

Figure 6: Anti-swing control strategy.

The proposed control strategy is implemented for a 3-dimensional case using three actuators. Howerever, more actuators may be used in combination with redundancy optimization, see Kjelland et al. (2012).

\section{System Modeling}

A dynamic model of the crane has been made in MATLAB Simscape ${ }^{\mathrm{TM}}$. 3D CAD models have been imported into the model using the Multibody library, and the hydraulic system has been modeled using the hydraulic library. A side view of the crane in the simulation model is shown in Figure 7.

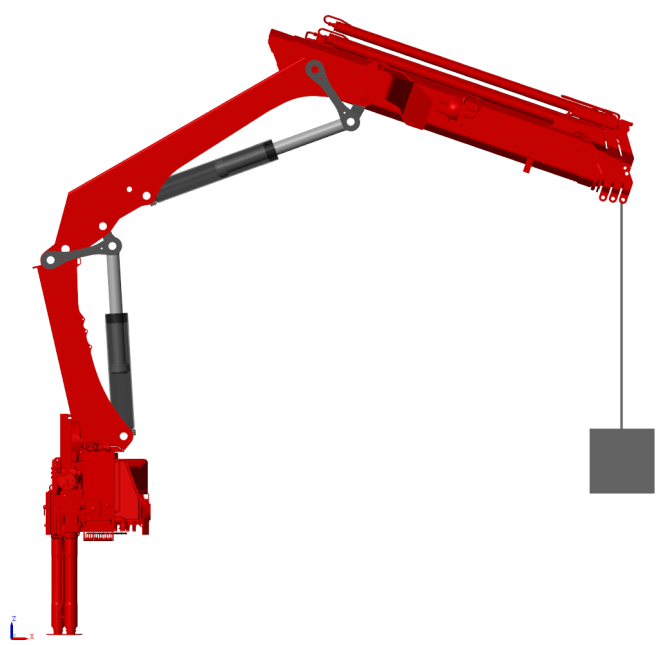

Figure 7: 2D view of the crane model in MATLAB Simscapce $^{\mathrm{TM}}$.

\subsection{Hanging Load Dynamics}

To derive the equations of motion for the hanging load, the Euler-Lagrange equations are used. For the following equations, the notation $s_{\alpha}=\sin (\alpha), c_{\alpha}=\cos (\alpha)$ is used. With the boom tip position defined as $\mathbf{r}_{t}=$ $\left[\begin{array}{lll}x_{t} & y_{t} & z_{t}\end{array}\right]^{T}$, the payload position is calculated as follows.

$$
\mathbf{r}_{p}=\mathbf{r}_{t}+L_{w}\left[\begin{array}{c}
s_{\alpha} \\
c_{\alpha} s_{\beta} \\
-c_{\alpha} c_{\beta}
\end{array}\right]
$$

The payload velocity is calculated by taking the time derivative of the payload position.

$$
\dot{\mathbf{r}}_{p}=\dot{\mathbf{r}}_{t}+L_{w}\left[\begin{array}{c}
\dot{\alpha} c_{\alpha} \\
\dot{\beta} c_{\alpha} c_{\beta}-\dot{\alpha} s_{\alpha} s_{\beta} \\
\dot{\alpha} s_{\alpha} c_{\beta}+\dot{\beta} c_{\alpha} s_{\beta}
\end{array}\right]+\dot{L}_{w}\left[\begin{array}{c}
s_{\alpha} \\
c_{\alpha} s_{\beta} \\
-c_{\alpha} c_{\beta}
\end{array}\right]
$$

The Lagrangian $\mathcal{L}$ of the system is defined as the kinetic energy $\mathcal{K}$ minus the potential energy $\mathcal{P}$, and is shown in Equation (3).

$$
\mathcal{L}=\mathcal{K}-\mathcal{P}
$$

The kinetic energy of the payload is:

$$
\mathcal{K}=\frac{1}{2} m_{p} \dot{\mathbf{r}}_{p}^{T} \dot{\mathbf{r}}_{p}
$$

The potential energy of the payload is:

$$
\mathcal{P}=m_{p} g\left(z_{t}-L_{w} c_{\alpha} c_{\beta}\right)
$$

Selecting the coordinate $q=\left[\begin{array}{ll}\alpha & \beta\end{array}\right]^{T}$, the EulerLagrange equation is defined in Equation 6.

$$
\frac{d}{d t} \frac{\partial \mathcal{L}}{\partial \dot{q}}-\frac{\partial \mathcal{L}}{\partial q}=0
$$

Solving Equation (6) gives two coupled second order differential equations, shown in Equation (7) and (8). The full calculations are given in Appendix A in Equations (A.1)-(A.13).

$$
\begin{aligned}
\ddot{\alpha}= & \frac{1}{L_{w}}\left(-c_{\alpha} \ddot{x}_{t}+s_{\alpha} s_{\beta} \ddot{y}_{t}-s_{\alpha} c_{\beta} \ddot{z}_{t}-2 \dot{\alpha} \dot{L}_{w}\right. \\
& \left.-s_{\alpha} c_{\alpha} \dot{\beta}^{2} L_{w}-g s_{\alpha} c_{\beta}\right) \\
\ddot{\beta}= & \frac{1}{c_{\alpha} L_{w}}\left(-c_{\beta} \ddot{y}_{t}-s_{\beta} \ddot{z}_{t}+2 s_{\alpha} \dot{\alpha} \dot{\beta} L_{w}\right. \\
& \left.-2 \dot{\beta} \dot{L}_{w}-g s_{\beta}\right)
\end{aligned}
$$

\subsection{Joint Space Kinematics}

The joint space kinematics describes the relation between the joint angles and Cartesian coordinates of 
the crane tip. Figure 8 shows the geometry which is used with the Denavit-Hartenberg parameters, where both booms are horizontal. The distances between consecutive joints are shown in Table 2. The DenavitHartenberg parameters are shown in Table 3, where $\mathbf{R}$ and $\mathbf{T}$ are rotational and translational matrices, respectively. The angles $\theta_{s}, \theta_{m}$, and $\theta_{k}$ denote the rotation about the slew joint, main joint, and knuckle joint, respectively.

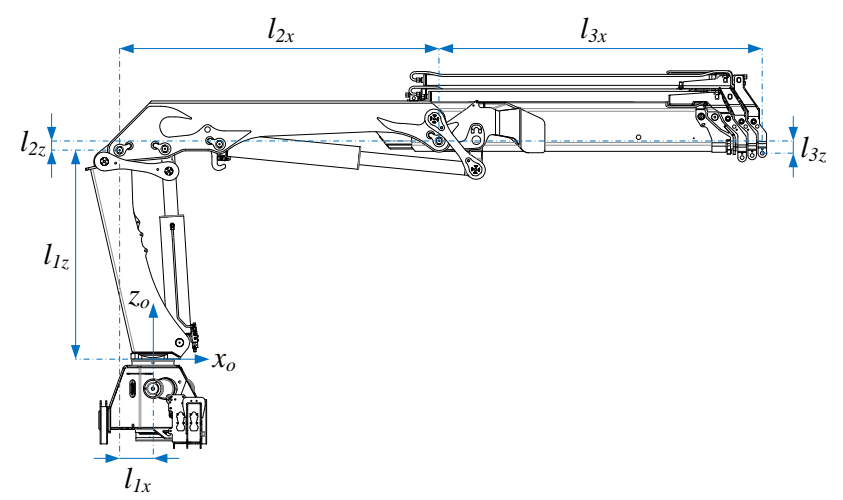

Figure 8: Crane geometry used with DenavitHartenberg parameters.

Table 2: Coordinates shown in Figure 8.

\begin{tabular}{ll}
\hline Name & Length $[\mathrm{m}]$ \\
\hline$l_{1 x}$ & 0.250 \\
$l_{1 z}$ & 1.569 \\
$l_{2 x}$ & 2.400 \\
$l_{2 z}$ & 0.070 \\
$l_{3 x}$ & 2.429 \\
$l_{3 z}$ & 0.093 \\
\hline
\end{tabular}

Table 3: Denavit-Hartenberg parameters.

\begin{tabular}{llll}
\hline $\mathbf{R}_{z}$ & $\mathbf{T}_{z}$ & $\mathbf{T}_{x}$ & $\mathbf{R}_{x}$ \\
\hline$\theta_{s}$ & $l_{1 z}$ & $-l_{1 x}$ & $90^{\circ}$ \\
$\theta_{m}$ & 0 & 0 & $-90^{\circ}$ \\
0 & $l_{2 z}$ & $l_{2 x}$ & $90^{\circ}$ \\
$\theta_{k}$ & 0 & 0 & $-90^{\circ}$ \\
0 & $-l_{3 z}$ & $l_{3 x}$ & 0 \\
\hline
\end{tabular}

The transformation matrix $\mathbf{A}_{D H}$ from the base of the crane to the tip of the crane can be established as a sequence of transformations based on the DenavitHartenberg parameters, shown in Equation (9).

$$
\begin{aligned}
\mathbf{A}_{D H}= & \mathbf{R}_{z}\left(\theta_{s}\right) \mathbf{T}_{z}\left(l_{1 z}\right) \mathbf{T}_{x}\left(-l_{1 x}\right) \mathbf{R}_{x}\left(90^{\circ}\right) \mathbf{R}_{z}\left(\theta_{m}\right) \\
& \mathbf{R}_{x}\left(-90^{\circ}\right) \mathbf{T}_{z}\left(l_{2 z}\right) \mathbf{T}_{x}\left(l_{2 x}\right) \mathbf{R}_{x}\left(90^{\circ}\right) \\
& \mathbf{R}_{z}\left(\theta_{k}\right) \mathbf{R}_{x}\left(-90^{\circ}\right) \mathbf{T}_{z}\left(-l_{3 z}\right) \mathbf{T}_{x}\left(l_{3 x}\right)
\end{aligned}
$$

The final matrix $\mathbf{A}_{D H}$ is shown in Equation (10). The joint kinematics from the crane base to the crane tip is now contained in $x_{t}, y_{t}$, and $z_{t}$.

$$
\begin{aligned}
\mathbf{A}_{D H}= & {\left[\begin{array}{cccc}
c_{\theta_{s}} c_{\theta_{m}+\theta_{k}} & -s_{\theta_{s}} & -c_{\theta_{s}} s_{\theta_{m}+\theta_{k}} & x_{t} \\
s_{\theta_{s}} c_{\theta_{m}+\theta_{k}} & c_{\theta_{s}} & -s_{\theta_{s}} s_{\theta_{m}+\theta_{k}} & y_{t} \\
s_{\theta_{m}+\theta_{k}} & 0 & c_{\theta_{m}+\theta_{k}} & z_{t} \\
0 & 0 & 0 & 1
\end{array}\right] } \\
x_{t}= & c_{\theta_{s}}\left(-l_{1 x}+l_{2 x} c_{\theta_{m}}-l_{2 z} s_{\theta_{m}}\right. \\
& \left.+l_{3 x} c_{\theta_{m}+\theta_{k}}+l_{3 z} s_{\theta_{m}+\theta_{k}}\right) \\
y_{t}= & s_{\theta_{s}}\left(-l_{1 x}+l_{2 x} c_{\theta_{m}}-l_{2 z} s_{\theta_{m}}\right. \\
& \left.+l_{3 x} c_{\theta_{m}+\theta_{k}}+l_{3 z} s_{\theta_{m}+\theta_{k}}\right) \\
z_{t}= & l_{1 z}+l_{2 x} s_{\theta_{m}}+l_{2 z} c_{\theta_{m}} \\
& +l_{3 x} s_{\theta_{m}+\theta_{k}}-l_{3 z} c_{\theta_{m}+\theta_{k}}
\end{aligned}
$$

To find the correlation between the desired crane tip velocities and the joint velocities, the inverse Jacobian matrix must be defined. The correlation between crane tip velocities and joint velocities is shown in Equations (14) and (15).

$$
\begin{gathered}
{\left[\begin{array}{c}
\dot{x}_{t} \\
\dot{y}_{t} \\
\dot{z}_{t}
\end{array}\right]=\mathbf{J}\left[\begin{array}{c}
\dot{\theta}_{s} \\
\dot{\theta}_{m} \\
\dot{\theta}_{k}
\end{array}\right]} \\
{\left[\begin{array}{c}
\dot{\theta}_{s} \\
\dot{\theta}_{m} \\
\dot{\theta}_{k}
\end{array}\right]=\mathbf{J}^{-1}\left[\begin{array}{c}
\dot{x}_{t} \\
\dot{y}_{t} \\
\dot{z}_{t}
\end{array}\right]}
\end{gathered}
$$

First, the Jacobian matrix is defined as the partial derivative of the crane tip position with respect to the joint angles, shown in Equation (16).

$$
\mathbf{J}=\left[\begin{array}{lll}
\frac{\partial}{\partial \theta_{s}}\left(x_{t}\right) & \frac{\partial}{\partial \theta_{m}}\left(x_{t}\right) & \frac{\partial}{\partial \theta_{k}}\left(x_{t}\right) \\
\frac{\partial}{\partial \theta_{s}}\left(y_{t}\right) & \frac{\partial}{\partial \theta_{m}}\left(y_{t}\right) & \frac{\partial}{\partial \theta_{k}}\left(y_{t}\right) \\
\frac{\partial}{\partial \theta_{s}}\left(z_{t}\right) & \frac{\partial}{\partial \theta_{m}}\left(z_{t}\right) & \frac{\partial}{\partial \theta_{k}}\left(z_{t}\right)
\end{array}\right]
$$




$$
\begin{aligned}
\frac{\partial}{\partial \theta_{s}}\left(x_{t}\right)= & -s_{\theta_{s}}\left(-l_{1 x}+l_{2 x} c_{\theta_{m}}-l_{2 z} s_{\theta_{m}}\right. \\
& \left.+l_{3 x} c_{\theta_{m}+\theta_{k}}+l_{3 z} s_{\theta_{m}+\theta_{k}}\right) \\
\frac{\partial}{\partial \theta_{m}}\left(x_{t}\right)= & c_{\theta_{s}}\left(-l_{2 x} s_{\theta_{m}}-l_{2 z} c_{\theta_{m}}\right. \\
& \left.-l_{3 x} s_{\theta_{m}+\theta_{k}}+l_{3 z} c_{\theta_{m}+\theta_{k}}\right) \\
\frac{\partial}{\partial \theta_{k}}\left(x_{t}\right)= & c_{\theta_{s}}\left(-l_{3 x} s_{\theta_{m}}+\theta_{k}+l_{3 z} c_{\theta_{m}+\theta_{k}}\right) \\
\frac{\partial}{\partial \theta_{s}}\left(y_{t}\right)= & c_{\theta_{s}}\left(-l_{1 x}+l_{2 x} c_{\theta_{m}}-l_{2 z} s_{\theta_{m}}\right. \\
& \left.+l_{3 x} c_{\theta_{m}+\theta_{k}}+l_{3 z} s_{\theta_{m}+\theta_{k}}\right) \\
\frac{\partial}{\partial \theta_{m}}\left(y_{t}\right)= & s_{\theta_{s}}\left(-l_{2 x} s_{\theta_{m}}-l_{2 z} c_{\theta_{m}}\right. \\
& \left.-l_{3 x} s_{\theta_{m}+\theta_{k}}+l_{3 z} c_{\theta_{m}+\theta_{k}}\right) \\
\frac{\partial}{\partial \theta_{k}}\left(y_{t}\right)= & s_{\theta_{s}}\left(-l_{3 x} s_{\theta_{m}}+\theta_{k}+l_{3 z} c_{\theta_{m}+\theta_{k}}\right) \\
\frac{\partial}{\partial \theta_{s}}\left(z_{t}\right)= & 0 \\
\frac{\partial}{\partial \theta_{m}}\left(z_{t}\right)= & l_{2 x} c_{\theta_{m}}-l_{2 z} s_{\theta_{m}} \\
& +l_{3 x} c_{\theta_{m}+\theta_{k}}+l_{3 z} s_{\theta_{m}+\theta_{k}} \\
\frac{\partial}{\partial \theta_{k}}\left(z_{t}\right)= & -l_{3 x} c_{\theta_{m}+\theta_{k}}-l_{3 z} s_{\theta_{m}+\theta_{k}} \\
&
\end{aligned}
$$

The inverse Jacobian matrix is used to generate the joint velocities, shown in Equations (26)-(38).

$$
\begin{aligned}
& \mathbf{J}^{\dagger} \triangleq \mathbf{J}^{-1}=\left[\begin{array}{lll}
J_{11}^{\dagger} & J_{12}^{\dagger} & J_{13}^{\dagger} \\
J_{21}^{\dagger} & J_{22}^{\dagger} & J_{23}^{\dagger} \\
J_{31}^{\dagger} & J_{32}^{\dagger} & J_{33}^{\dagger}
\end{array}\right] \\
& \dot{\theta}_{s}=J_{11}^{\dagger} \dot{x}_{t}+J_{12}^{\dagger} \dot{y}_{t}+J_{13}^{\dagger} \dot{z}_{t} \\
& \dot{\theta}_{m}=J_{21}^{\dagger} \dot{x}_{t}+J_{22}^{\dagger} \dot{y}_{t}+J_{23}^{\dagger} \dot{z}_{t} \\
& \dot{\theta}_{k}=J_{31}^{\dagger} \dot{x}_{t}+J_{32}^{\dagger} \dot{y}_{t}+J_{33}^{\dagger} \dot{z}_{t} \\
& J_{11}^{\dagger}=\frac{-s_{\theta_{s}}}{-l_{1 x}+l_{2 x} c_{\theta_{m}}-l_{2 z} s_{\theta_{m}}+l_{3 x} c_{\theta_{m}+\theta_{k}}+l_{3 z} s_{\theta_{m}+\theta_{k}}} \\
& J_{12}^{\dagger}=\frac{c_{\theta_{s}}}{-l_{1 x}+l_{2 x} c_{\theta_{m}}-l_{2 z} s_{\theta_{m}}+l_{3 x} c_{\theta_{m}+\theta_{k}}+l_{3 z} s_{\theta_{m}+\theta_{k}}} \\
& J_{13}^{\dagger}=0 \\
& J_{21}^{\dagger}=\frac{-c_{\theta_{s}}\left(l_{3 x} c_{\theta_{m}+\theta_{k}}+l_{3 z} s_{\theta_{m}+\theta_{k}}\right)}{-l_{2 x} l_{3 x} s_{\theta_{k}}+l_{2 x} l_{3 z} c_{\theta_{k}}+l_{2 z} l_{3 x} c_{\theta_{k}}+l_{2 z} l_{3 z} s_{\theta_{k}}} \\
& J_{22}^{\dagger}=\frac{-s_{\theta_{s}}\left(l_{3 x} c_{\theta_{m}+\theta_{k}}+l_{3 z} s_{\theta_{m}+\theta_{k}}\right)}{-l_{2 x} l_{3 x} s_{\theta_{k}}+l_{2 x} l_{3 z} c_{\theta_{k}}+l_{2 z} l_{3 x} c_{\theta_{k}}+l_{2 z} l_{3 z} s_{\theta_{k}}} \\
& J_{23}^{\dagger}=\frac{-l_{3 x} s_{\theta_{m}+\theta_{k}}+l_{3 z} c_{\theta_{m}+\theta_{k}}}{-l_{2 x} l_{3 x} s_{\theta_{k}}+l_{2 x} l_{3 z} c_{\theta_{k}}+l_{2 z} l_{3 x} c_{\theta_{k}}+l_{2 z} l_{3 z} s_{\theta_{k}}} \\
& J_{31}^{\dagger}=\frac{c_{\theta_{s}}\left(l_{2 x} c_{\theta_{m}}-l_{2 z} s_{\theta_{m}}+l_{3 x} c_{\theta_{m}+\theta_{m}}+l_{3 z} s_{\theta_{m}+\theta_{k}}\right)}{-l_{2 x} l_{3 x} s_{\theta_{k}}+l_{2 x} l_{3 z} c_{\theta_{k}}+l_{2 z} l_{3 x} c_{\theta_{k}}+l_{2 z} l_{3 z} s_{\theta_{k}}} \\
& J_{32}^{\dagger}=\frac{s_{\theta_{s}}\left(l_{2 x} c_{\theta_{m}}-l_{2 z} s_{\theta_{m}}+l_{3 x} c_{\theta_{m}+\theta_{m}}+l_{3 z} s_{\theta_{m}+\theta_{k}}\right)}{-l_{2 x} l_{3 x} s_{\theta_{k}}+l_{2 x} l_{3 z} c_{\theta_{k}}+l_{2 z} l_{3 x} c_{\theta_{k}}+l_{2 z} l_{3 z} s_{\theta_{k}}} \\
& J_{33}^{\dagger}=\frac{l_{2 x} s_{\theta_{m}}+l_{2 z} c_{\theta_{m}}+l_{3 x} s_{\theta_{m}+\theta_{k}}-l_{3 z} c_{\theta_{m}+\theta_{k}}}{-l_{2 x} l_{3 x} s_{\theta_{k}}+l_{2 x} l_{3 z} c_{\theta_{k}}+l_{2 z} l_{3 x} c_{\theta_{k}}+l_{2 z} l_{3 z} s_{\theta_{k}}}
\end{aligned}
$$

\subsection{Actuator Space Kinematics}

The actuator space kinematics describes the relation between the cylinder lengths, cylinder velocities, joint angles, and joint angular velocities. They have previously been developed in Jensen et al. (2021). The shorthand kinematic expressions are shown in Equations (39)-(47). Figure 9 and Figure 10 show the geometry of the linkage system for the main joint and knuckle joint, respectively.

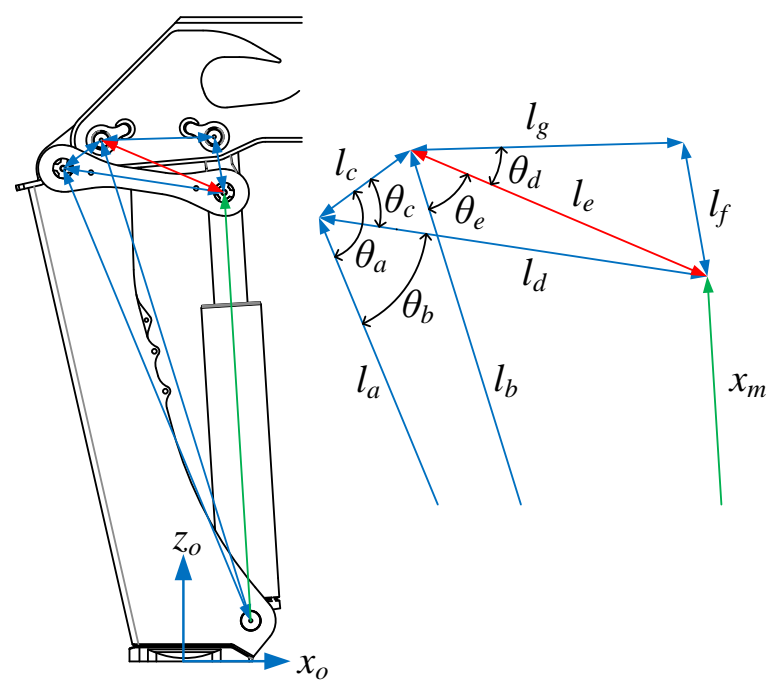

Figure 9: Geometry of the linkage system for the main joint.

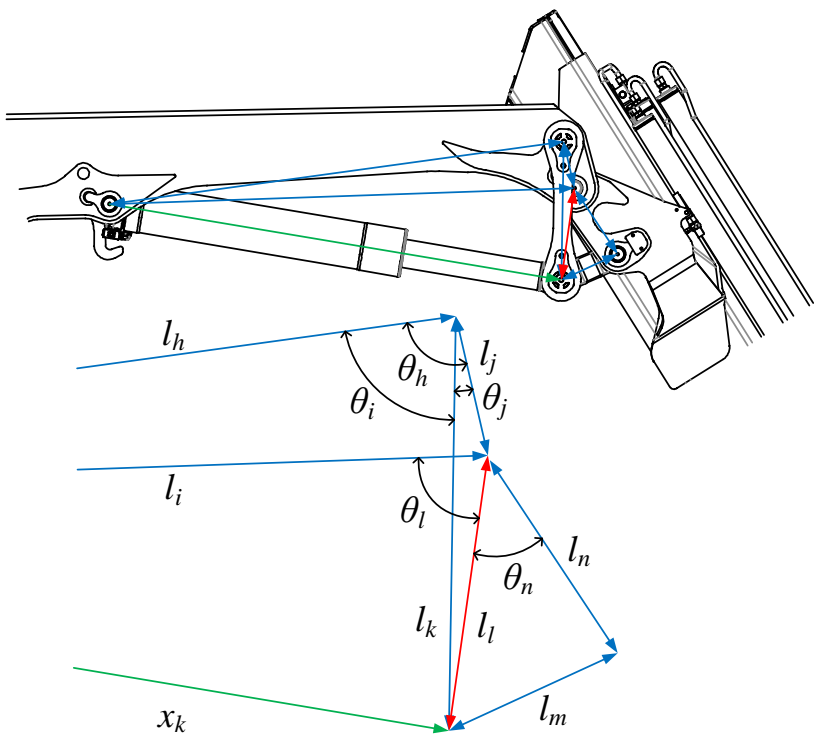

Figure 10: Geometry of the linkage system for the knuckle joint. 


$$
\begin{aligned}
\theta_{s} & =\frac{x_{s}}{r_{s}} \\
\theta_{m} & =\theta_{m}\left(x_{m}\right) \\
\theta_{k} & =\theta_{k}\left(x_{k}\right) \\
\dot{\theta}_{s} & =\frac{\dot{x}_{s}}{r_{s}} \\
\dot{\theta}_{m} & =\frac{\partial \theta_{m}\left(x_{m}\right)}{\partial x_{m}} \dot{x}_{m} \\
\dot{\theta}_{k} & =\frac{\partial \theta_{k}\left(x_{k}\right)}{\partial x_{k}} \dot{x}_{k} \\
\dot{x}_{s} & =\dot{\theta}_{s} r_{s} \\
\dot{x}_{m} & =\left(\frac{\partial \theta_{m}\left(x_{m}\right)}{\partial x_{m}}\right)^{-1} \dot{\theta}_{m} \\
& =\theta_{x_{m}}^{\dagger} \dot{\theta}_{m} \\
\dot{x}_{k} & =\left(\frac{\partial \theta_{k}\left(x_{k}\right)}{\partial x_{k}}\right)^{-1} \dot{\theta}_{k} \\
& =\theta_{x_{k}}^{\dagger} \dot{\theta}_{k}
\end{aligned}
$$

\subsection{Hydraulic Modeling}

All three cylinders are controlled by pressure compensated directional valves. An illustration of the valve is shown in Figure 11. The pressure compensator senses the load pressure to keep the pressure drop over the directional valve constant, thus ensuring a load independent flow. The governing equations of the pressure compensator are given in Equations (48)-(50).

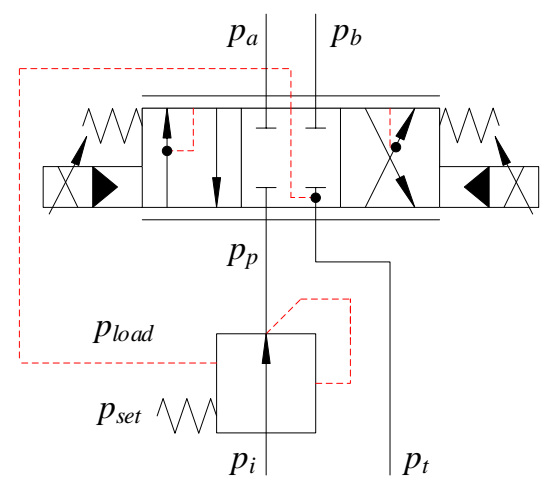

Figure 11: Pressure compensated directional valve.

$$
\begin{aligned}
u_{p c} & =\frac{p_{\text {set }}+p_{\text {load }}-p_{p}}{\Delta p_{c}} \\
p_{\text {load }} & = \begin{cases}p_{a} & \text { if } u_{\text {spool }} \geq 0 \\
p_{b} & \text { otherwise }\end{cases} \\
Q_{p c} & =k_{p c} u_{p c} \sqrt{p_{i}-p_{p}}
\end{aligned}
$$

where;

$$
\begin{array}{ll}
u_{p c} & =\text { opening of compensator, } 0 \leq u_{p c} \leq 1 \\
p_{p} & =\text { compensated pressure at port } p \\
\Delta p_{c} & =\text { pressure difference when fully opened } \\
p_{a} & =\text { pressure at port } a \\
p_{b} & =\text { pressure at port } b \\
p_{t} & =\text { tank pressure } \\
p_{\text {set }} & =\text { spring pressure setting } \\
p_{\text {load }} & =\text { load pressure } \\
u_{s p o o l} & =\text { main spool position, }-1 \leq u_{\text {spool }} \leq 1 \\
Q_{p c} & =\text { flow in pressure compensator } \\
k_{p c} & =\text { flow gain of compensator } \\
p_{i} & =\text { compensator inlet pressure }
\end{array}
$$

The steady state of $p_{p}$ is then given by Equation (51).

$$
p_{p}=p_{\text {load }}+p_{\text {set }}
$$

The sensing of the load pressures $p_{a}$ and $p_{b}$ ensures that the pressure drop over the directional control valve always equals $p_{\text {set }}$, and that the flow is load independent. This is shown in Equation (52).

$$
\begin{aligned}
Q & =k_{v} u_{\text {spool }} \sqrt{p_{p}-p_{\text {load }}} \\
& =k_{v} u_{\text {spool }} \sqrt{p_{\text {set }}} \\
& =Q_{\text {max }} u_{\text {spool }}
\end{aligned}
$$

where;

$$
\begin{aligned}
& k_{v}=\text { flow gain of the directional valve } \\
& Q_{\max }=\text { maximum valve flow }
\end{aligned}
$$

To assist with load holding, lowering of the load, and protection against pressure surges, counterbalance valves are used between the directional valve and the hydraulic cylinder. Figure 12 shows an illustration of double counterbalance valves, as used on the slew cylinder and knuckle cylinder. The main cylinder uses a single counterbalance valve.

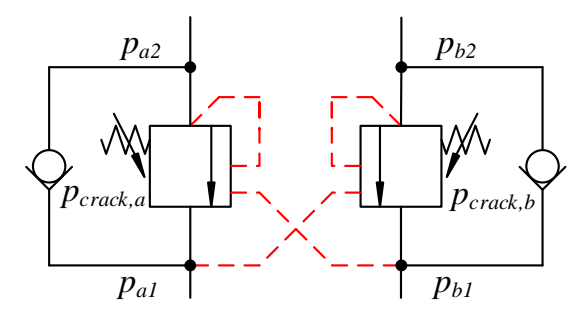

Figure 12: Double counterbalance valves.

The governing equations of the counterbalance valves are shown in Equations (53) and (54).

$$
\begin{aligned}
& u_{a}=\frac{p_{a 2}+\psi p_{b 1}-p_{\text {crack }, a}}{\Delta p_{C B V}} \\
& u_{b}=\frac{p_{b 2}+\psi p_{a 1}-p_{\text {crack }, b}}{\Delta p_{C B V}}
\end{aligned}
$$


where;

\begin{tabular}{|c|c|c|}
\hline$u_{a}$ & $=$ & opening of valve $a, 0 \leq u_{a} \leq 1$ \\
\hline$u_{b}$ & $=$ & opening of valve $b, 0 \leq u_{b} \leq 1$ \\
\hline$p_{a 1}$ & $=$ & pressure at valve $a$ input side \\
\hline$p_{a 2}$ & $=$ & pressure at valve $a$ actuator side \\
\hline$p_{b 1}$ & $=$ & pressure at valve $b$ input side \\
\hline$p_{b 2}$ & $=$ & pressure at valve $b$ actuator side \\
\hline$p_{\text {crack }, a}$ & $=$ & crack pressure of valve $a$ \\
\hline $\begin{array}{l}p_{\text {crack }, b} \\
\psi\end{array}$ & $\begin{array}{l}= \\
=\end{array}$ & $\begin{array}{l}\text { crack pressure of valve } b \\
\text { pilot area ratio }\end{array}$ \\
\hline$\Delta p_{C B V}$ & $=$ & pressure difference when fully opened \\
\hline
\end{tabular}

\section{Control System Design}

The control of the hydraulic cylinders uses feedback of the position error, and feedforward based on the velocity reference. Since the hydraulic system yields load independent velocity control, feedforward is an effective control method, as stated in Bak and Hansen (2013) and Jensen et al. (2020b). The anti-swing gain $k_{a}$ and the payload angles $\alpha$ and $\beta$ are used to generate two anti-swing crane tip velocities, $\dot{x}_{t, a}$ and $\dot{y}_{t, a}$ in order to suppress the payload angles. These velocities are transformed into joint space and then into actuator space, to yield the anti-swing slew velocity $\dot{\theta}_{s, a}$ and cylinder velocities $\dot{x}_{m, a}$ and $\dot{x}_{k, a}$. This is shown in Equations (55)-(58).

The full control system is shown in Figure 13, outlining the feedback controller (blue), feedforward controller (red), and anti-swing controller (green). It should be noted that the slew angle is used instead of the slew cylinder length since it is connected to a sensor on the crane. The anti-swing controller uses actuator kinematics (Act. Kin.), inverse Jacobian (Inv. Jac.), and inverse actuator kinematics (Inv. Act.). Both the feedforward controller and anti-swing controller use the gain $k_{f f}$ to calculate the valve opening from actuator velocity.

$$
\begin{aligned}
{\left[\begin{array}{c}
\dot{x}_{t, a} \\
\dot{y}_{t, a} \\
\dot{z}_{t, a}
\end{array}\right] } & =k_{a}\left[\begin{array}{l}
\alpha \\
\beta \\
0
\end{array}\right] \\
{\left[\begin{array}{c}
\dot{\theta}_{s, a} \\
\dot{\theta}_{m, a} \\
\dot{\theta}_{k, a}
\end{array}\right] } & =J^{\dagger}\left[\begin{array}{l}
\dot{x}_{t, a} \\
\dot{y}_{t, a} \\
\dot{z}_{t, a}
\end{array}\right] \\
\dot{x}_{m, a} & =\theta_{x_{m}}^{\dagger} \dot{\theta}_{m, a} \\
\dot{x}_{k, a} & =\theta_{x_{k}}^{\dagger} \dot{\theta}_{k, a}
\end{aligned}
$$

The anti-swing cylinder velocities $\dot{x}_{m, a}$ and $\dot{x}_{k, a}$ are then multiplied by $k_{f f}$ to generate the valve opening. The control outputs for the control system are shown in Equations (59)-(61).

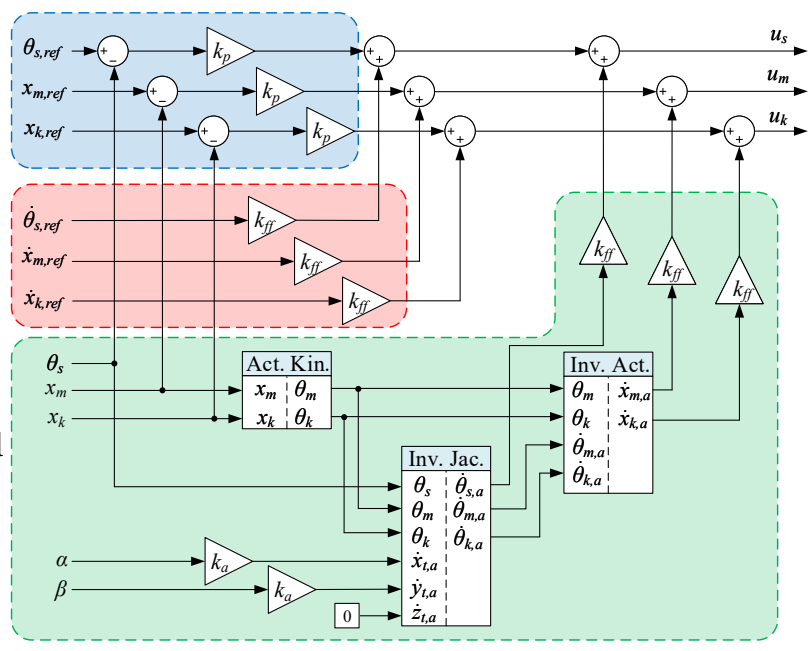

Figure 13: Illustration of the 3D anti-swing control system, with feedback controller (blue), feedforward controller (red), and anti-swing controller (green).

$$
\begin{aligned}
u_{s} & =\left(\theta_{s, r e f}-\theta_{s}\right) k_{p, s}+\left(\dot{\theta}_{s, r e f}+\dot{\theta}_{s, a}\right) k_{f f, s} \\
u_{m} & =\left(x_{m, r e f}-x_{m}\right) k_{p, m}+\left(\dot{x}_{m, r e f}+\dot{x}_{m, a}\right) k_{f f, m} \\
u_{k} & =\left(x_{k, r e f}-x_{k}\right) k_{p, k}+\left(\dot{x}_{k, r e f}+\dot{x}_{k, a}\right) k_{f f, k}
\end{aligned}
$$

\subsection{Theoretical closed loop analysis}

An analysis of the closed loop hanging load dynamics can be conducted based on the open loop hanging load dynamics and the selected control law. The control law controls the velocity of the crane tip. Recalling from earlier sections, the open loop dynamics and control law are given as:

$$
\begin{aligned}
\ddot{\alpha}= & \frac{1}{L_{w}}\left(-c_{\alpha} \ddot{x}_{t}+s_{\alpha} s_{\beta} \ddot{y}_{t}-s_{\alpha} c_{\beta} \ddot{z}_{t}-2 \dot{\alpha} \dot{L}_{w}\right. \\
& \left.-s_{\alpha} c_{\alpha} \dot{\beta}^{2} L_{w}-g s_{\alpha} c_{\beta}\right) \\
\ddot{\beta}= & \frac{1}{c_{\alpha} L_{w}}\left(-c_{\beta} \ddot{y}_{t}-s_{\beta} \ddot{z}_{t}+2 s_{\alpha} \dot{\alpha} \dot{\beta} L_{w}\right. \\
& \left.-2 \dot{\beta} \dot{L}_{w}-g s_{\beta}\right) \\
\dot{x}_{t}= & \alpha k_{a} \\
\dot{y}_{t}= & \beta k_{a} \\
\dot{z}_{t}= & 0
\end{aligned}
$$

The expressions for $\ddot{x}_{t}, \ddot{y}_{t}$ and $\ddot{z}_{t}$ can be made by taking the time derivative of the crane tip velocities:

$$
\begin{aligned}
\ddot{x}_{t} & =\dot{\alpha} k_{a} \\
\ddot{y}_{t} & =\dot{\beta} k_{a} \\
\ddot{z}_{t} & =0
\end{aligned}
$$


The closed loop hanging load dynamics can now be described as:

$$
\begin{aligned}
\ddot{\alpha}= & \frac{1}{L_{w}}\left(-c_{\alpha} \dot{\alpha} k_{a}+s_{\alpha} s_{\beta} \dot{\beta} k_{a}-2 \dot{\alpha} \dot{L}_{w}\right. \\
& \left.-s_{\alpha} c_{\alpha} \dot{\beta}^{2} L_{w}-g s_{\alpha} c_{\beta}\right) \\
\ddot{\beta}= & \frac{1}{c_{\alpha} L_{w}}\left(-c_{\beta} \dot{\beta} k_{a}+2 s_{\alpha} \dot{\alpha} \dot{\beta} L_{w}\right. \\
& \left.-2 \dot{\beta} \dot{L}_{w}-g s_{\beta}\right)
\end{aligned}
$$

Linearization is conducted to analyze the damping that the control law provides. Linearizing around $\alpha \approx \beta \approx 0$ and $\dot{L}_{w} \approx 0$ yields two decoupled equations of motion:

$$
\begin{aligned}
& \ddot{\alpha}=-\frac{\dot{\alpha} k_{a}}{L_{w}}-\frac{g \alpha}{L_{w}} \\
& \ddot{\beta}=-\frac{\dot{\beta} k_{a}}{L_{w}}-\frac{g \beta}{L_{w}}
\end{aligned}
$$

A Laplace transform is performed on the linearized and decoupled equations of motion, yielding:

$$
\begin{aligned}
& s^{2} \alpha=-\frac{s \alpha k_{a}}{L_{w}}-\frac{g \alpha}{L_{w}} \\
& s^{2} \beta=-\frac{s \beta k_{a}}{L_{w}}-\frac{g \beta}{L_{w}}
\end{aligned}
$$

The two equations yield the same second order transfer function, given as:

$$
\begin{aligned}
s^{2}+\frac{s k_{a}}{L_{w}}+\frac{g}{L_{w}} & =0 \\
s^{2}+2 s \zeta \omega+\omega^{2} & =0
\end{aligned}
$$

The bandwidth and damping ratio are calculated as:

$$
\begin{aligned}
& \omega=\sqrt{\frac{g}{L_{w}}} \\
& \zeta=\frac{k_{a}}{2 \sqrt{L_{w} g}}
\end{aligned}
$$

It can be seen that the damping ratio $\zeta$ increases as the anti-swing gain $k_{a}$ increases, and that the system is stable with $k_{a}>0$, yielding poles in the left halfplane. An analytical expression for the anti-swing gain can now be calculated based on desired damping ratio and wire length by rearranging Equation (79).

$$
k_{a}=2 \zeta \sqrt{L_{w} g}
$$

Equation (80) can be used to achieve the desired damping of the hanging payload and for the purpose of gain scheduling for systems with a variable wire length $L_{w}$.

\section{System Simulation}

For the simulation, a path controller based on a trapezoidal velocity reference is used, as described in Jensen et al. (2020b). The path controller operates in actuator space, and uses segments of constant cylinder velocity. The position reference for each actuator is shown in Figure 14. The relevant parameters for the simulation are shown in Table 4.

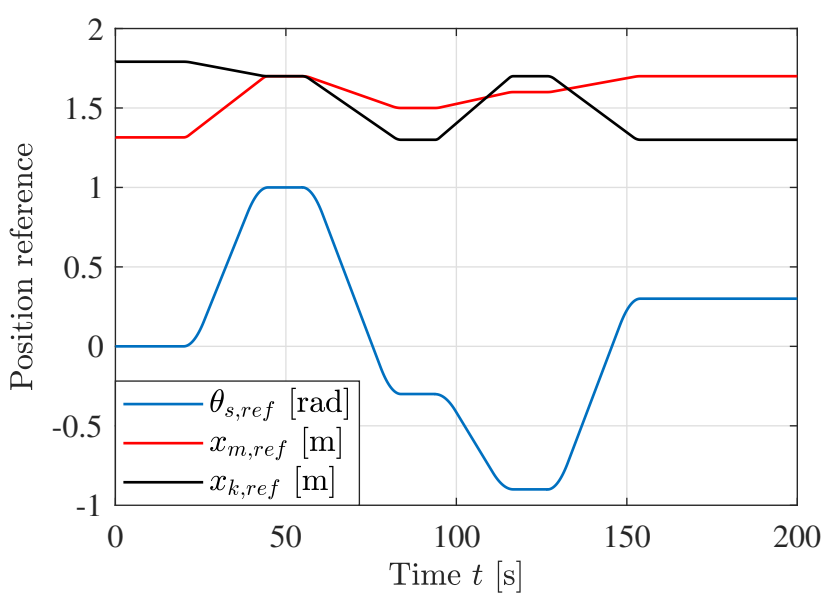

Figure 14: Position reference for each actuator.

Table 4: Simulation parameters.

\begin{tabular}{lll}
\hline Description & Name & Value \\
\hline Slew feedback & $k_{p, s}$ & $1 \mathrm{rad}^{-1}$ \\
Slew feedforward & $k_{f f, s}$ & $8.84 \mathrm{~s} / \mathrm{rad}$ \\
Main feedback & $k_{p, m}$ & $5 \mathrm{~m}^{-1}$ \\
Main out-stroke feedforward & $k_{f f, m}^{+}$ & $30.16 \mathrm{~s} / \mathrm{m}$ \\
Main in-stroke feedforward & $k_{f f, m}^{-}$ & $18.37 \mathrm{~s} / \mathrm{m}$ \\
Knuckle feedback & $k_{p, k}$ & $20 \mathrm{~m}^{-1}$ \\
Knuckle out-stroke feedforward & $k_{f f, k}^{+}$ & $26.51 \mathrm{~s} / \mathrm{m}$ \\
Knuckle in-stroke feedforward & $k_{f f, k}^{-}$ & $14.72 \mathrm{~s} / \mathrm{m}$ \\
Wire length & $L_{w}$ & $2 \mathrm{~m}$ \\
Anti-swing gain & $k_{a}$ & $5 \mathrm{~m} / \mathrm{s}$ \\
\hline
\end{tabular}

A plot of the swing angles $\alpha$ and $\beta$ without control is shown in Figure 15. The pendulum is oscillating heavily as expected. A plot of the same angles with anti-swing control is shown in Figure 16. Although both angles are lower, the angle $\beta$ experiences high frequency oscillations. The source of the oscillations are identified as the slewing motion itself. Looking at the slew position error, the high frequency oscillations occur both with and without anti-swing control, shown in Figure 17. 


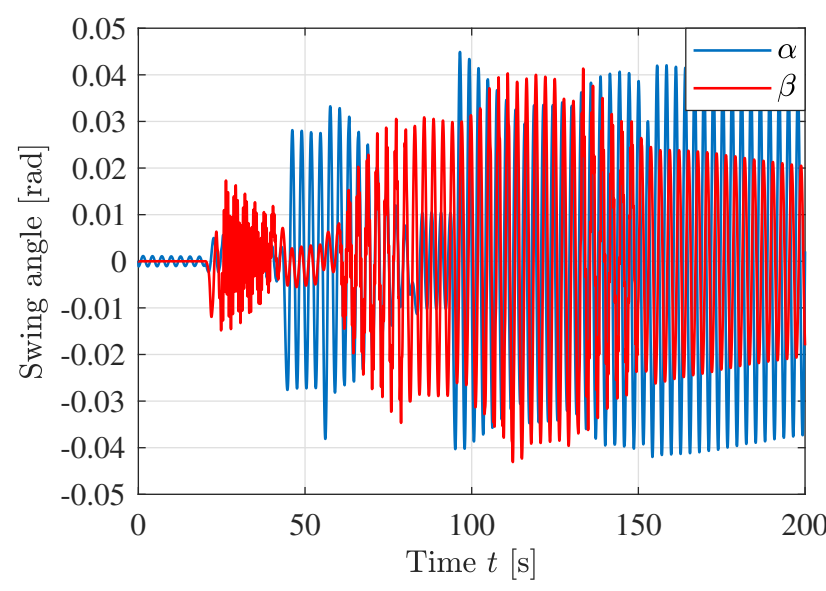

Figure 15: Swing angles without anti-swing control.

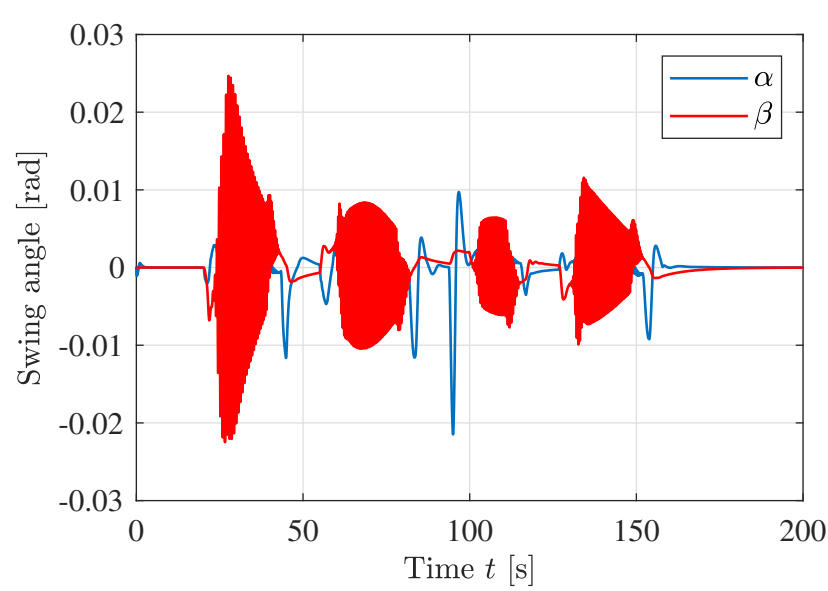

Figure 16: Swing angles with anti-swing control.

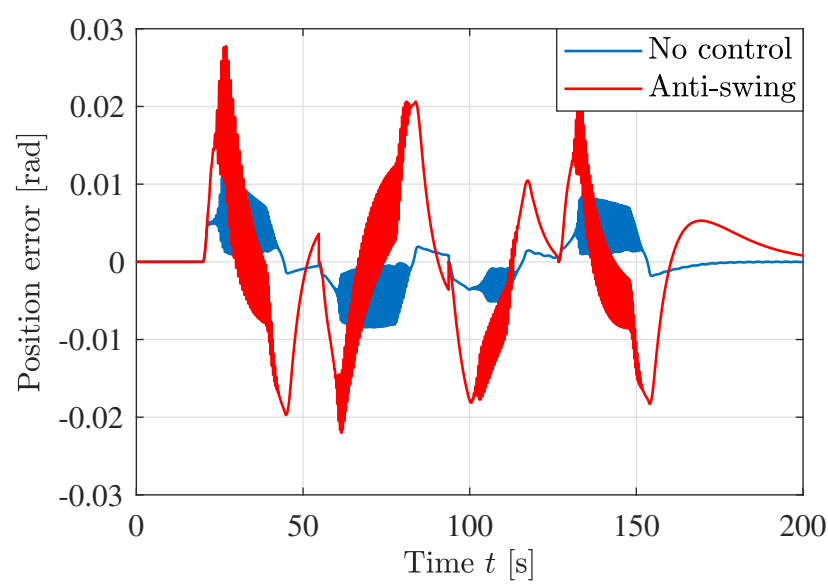

Figure 17: Slew position error with and without antiswing control.
To compensate for the oscillations in the slewing motion, pressure feedback is implemented. By measuring the load pressure, the control signal is modified by subtracting the high-pass filtered load pressure. Pressure feedback has previously been used to dampen oscillations of hydraulic systems, see Pedersen et al. (2016), Pedersen and Andersen (2018) and Hagen et al. (2019). The implementation is shown in Equation (81), using the filter gain $k_{p f}$, filter frequency $\omega_{p f}$, and load pressure $p_{L}$. Figure 18 shows the swing angles with antiswing and pressure feedback, with $k_{p f}=0.02$ bar $^{-1}$ and $\omega_{p f}=15 \mathrm{rad} / \mathrm{s}$. The high frequency oscillations in the swing angle $\beta$ are successfully suppressed, and both angles are significantly dampened compared to the system without anti-swing control. The position error with anti-swing and pressure feedback is shown in Figure 19, showing no oscillations.

$$
\hat{u}_{s}=u_{s}-\frac{k_{p f} s}{s+\omega_{p f}} p_{L}
$$

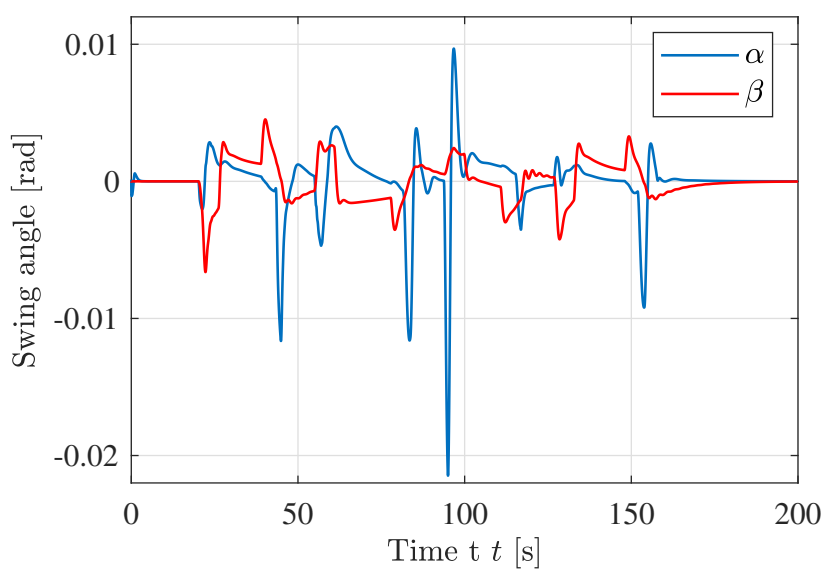

Figure 18: Swing angles with anti-swing and pressure feedback. 


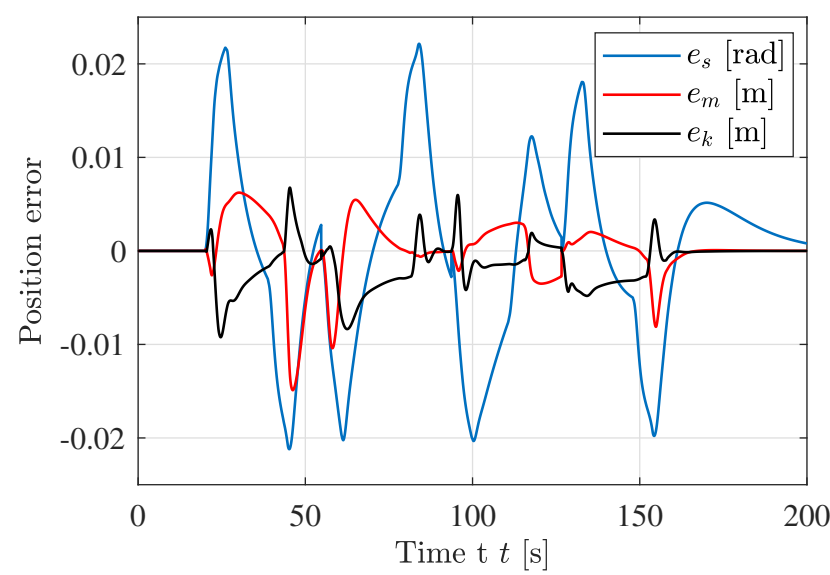

Figure 19: Position error with anti-swing and pressure feedback.

To evaluate the performance of the anti-swing controller and pressure feedback, a quantitative analysis is performed comparing the RMS value of the three position errors and two swing angles. Table 5 shows the RMS values when the system is simulated without anti-swing control, with anti-swing control (AS), and with both anti-swing control and pressure feedback $(\mathrm{AS}+\mathrm{PF})$. Due to the increase in the three position errors, it is clear that the anti-swing controller has a negative impact. This is expected and tolerated, since the primary focus of the control system is to suppress the swing angles. The pressure feedback also gives a negative impact on the position error. However, looking at the slew position error $e_{s}$ from Figure 17 and 19, the elimination of the high frequency oscillations is a big advantage. For the hanging payload, both the antiswing control and the pressure feedback give a large reduction in the swing angles, with a decrease in the RMS value of approximately $90 \%$. The introduction of the pressure feedback yields a significant improvement for the angle $\beta$, which is also seen in Figure 16 and 18.

Table 5: Quantitative simulation results.

\begin{tabular}{llll}
\hline Variable & No control & AS & AS+PF \\
\hline $\operatorname{RMS}(\alpha)[\mathrm{mrad}]$ & 21.21 & 2.55 & 2.52 \\
$\operatorname{RMS}(\beta)[\mathrm{mrad}]$ & 16.97 & 4.25 & 1.47 \\
$\operatorname{RMS}\left(e_{s}\right)[\mathrm{mrad}]$ & 3.17 & 9.03 & 9.73 \\
$\operatorname{RMS}\left(e_{m}\right)[\mathrm{mm}]$ & 0.35 & 3.21 & 3.27 \\
$\operatorname{RMS}\left(e_{k}\right)[\mathrm{mm}]$ & 1.54 & 2.79 & 2.78 \\
\hline
\end{tabular}

\section{Experimental Results}

The anti-swing controller is implemented on a CompactRIO connected to the HMF 2020K4 loader crane.
A picture of the test setup is shown in Figure 20. The sensor used in the experiments is the BNO055 Absolute Orientation Sensor from Bosch Sensortec. It outputs three Euler angles and they are all used to calculate the payload angles $\alpha$ and $\beta$.

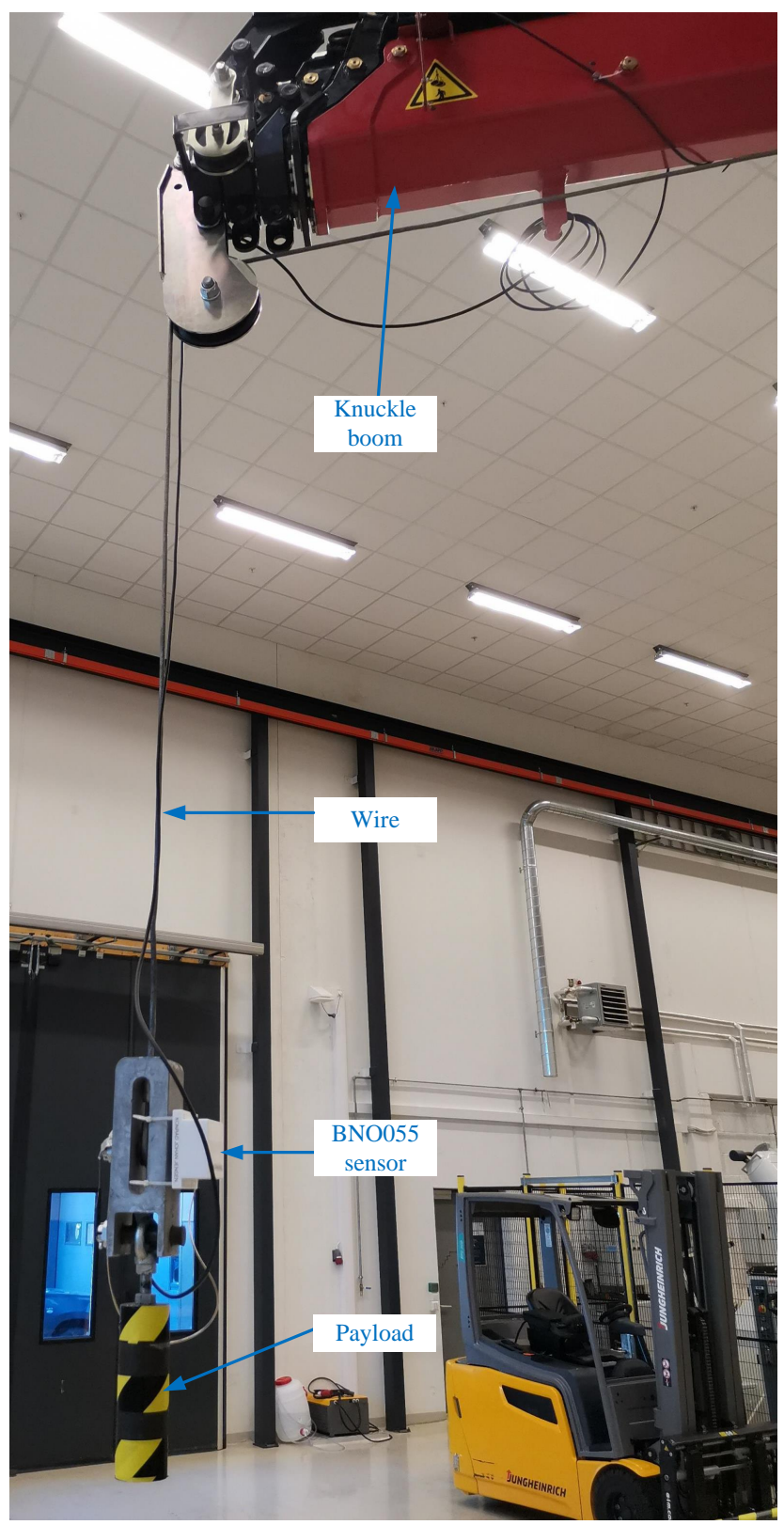

Figure 20: HMF 2020K4 crane in the laboratory.

There is some deadband in the valves on the HMF 2020K4 loader crane, and therefore deadband compensation has been implemented for the laboratory experiments. The identified deadbands for the valves are shown in Table 6 . The equation for the deadband compensation is shown in Equation (82). By introducing a small transition region $\tilde{u}$, the compensated control 
signal will be continuous which avoids unnecessary oscillations of the spool, while ensuring that the valve will be able to stay closed when no movement is needed.

Table 6: Identified deadband for the actuators.

\begin{tabular}{lll}
\hline Actuator & Out, $u^{+}$ & In, $u^{-}$ \\
\hline Slew & 0.22 & -0.26 \\
Main & 0.24 & -0.22 \\
Knuckle & 0.20 & -0.31 \\
\hline
\end{tabular}

$$
\hat{u}= \begin{cases}\min \left(u^{+}+\left(1-u^{+}\right) u, \frac{u^{+}}{\tilde{u}} u\right) & \text { if } u>0 \\ \max \left(u^{-}+\left(1+u^{-}\right) u,-\frac{u^{-}}{\tilde{u}} u\right) & \text { else }\end{cases}
$$

where;

$$
\begin{aligned}
& \hat{u}=\text { compensated control signal } \\
& u=\text { control signal } \\
& u^{+}=\text {out-stroke deadband } \\
& u^{-}=\text {in-stroke deadband } \\
& \tilde{u}=\text { transition region, } 0.01
\end{aligned}
$$

In the laboratory there was identified some drift in the payload angle sensor. This has been removed with a digital high pass filter, which is shown in Equations (83) and (84). This implementation was also used for the pressure feedback filter.

$$
\begin{aligned}
y_{i} & =k_{f} y_{i-1}+k_{f}\left(x_{i}-x_{i-1}\right) \\
k_{f} & =\frac{T_{f}}{T_{f}+T_{s}}
\end{aligned}
$$

where;

$$
\begin{aligned}
& i=\text { sample number } \\
& y=\text { filter output } \\
& x=\text { filter input } \\
& T_{f}=\text { filter time constant } \\
& T_{s}=\text { sample time, } 0.01 \mathrm{~s}
\end{aligned}
$$

To avoid filtering out the motion of the payload, the filter time constant $T_{f}$ should be larger than the pendulum period $T_{p}$. The pendulum period is calculated based on the wire length, shown in Equation (85). Because of the value of $T_{p}$ the filter time constant has been set to $T_{f}=3 \mathrm{~s}$.

$$
T_{p}=2 \pi \sqrt{\frac{L_{w}}{g}}=2.837 \mathrm{~s}
$$

Due to limited space, a path shorter than the one used in the simulations is used in the laboratory. The position reference used in the laboratory is shown in Figure 21. The parameters used in the laboratory are shown in Table 7. The slew feedback, slew feedforward, and main feedback have been adjusted to improve position tracking.

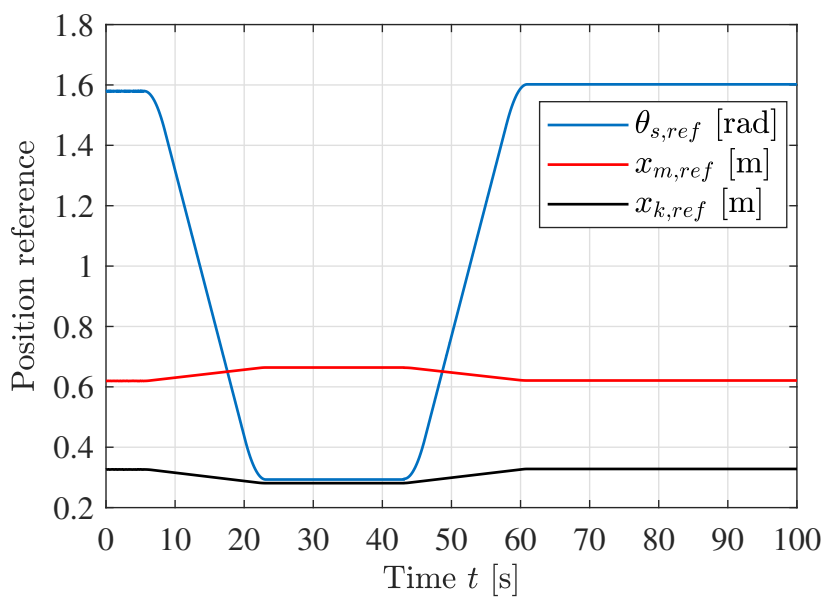

Figure 21: Position reference used in laboratory.

Table 7: Parameters used in laboratory.

\begin{tabular}{lll}
\hline Description & Name & Value \\
\hline Slew feedback & $k_{p, s}$ & $3 \mathrm{rad}^{-1}$ \\
Slew feedforward & $k_{f f, s}$ & $5.67 \mathrm{~s} / \mathrm{rad}$ \\
Main feedback & $k_{p, m}$ & $15 \mathrm{~m}^{-1}$ \\
Main out-stroke feedforward & $k_{f f, m}^{+}$ & $30.16 \mathrm{~s} / \mathrm{m}$ \\
Main in-stroke feedforward & $k_{f f, m}^{-}$ & $18.37 \mathrm{~s} / \mathrm{m}$ \\
Knuckle feedback & $k_{p, k}$ & $20 \mathrm{~m}^{-1}$ \\
Knuckle out-stroke feedforward & $k_{f f, k}^{+}$ & $26.51 \mathrm{~s} / \mathrm{m}$ \\
Knuckle in-stroke feedforward & $k_{f f, k}^{-}$ & $14.72 \mathrm{~s} / \mathrm{m}$ \\
Wire length & $L_{w}$ & $2 \mathrm{~m}$ \\
Anti-swing gain & $k_{a}$ & $5 \mathrm{~m} / \mathrm{s}$ \\
\hline
\end{tabular}

A plot of the swing angles without anti-swing control is shown in Figure 22, showing oscillations of the payload as expected. A plot of the position error without control is shown in Figure 23, showing good tracking except for the start of the slew actuator motion.

The influence of the pressure feedback turned out to be significant. The system became unstable without it, and proper tuning was needed to reach stability. The parameters used in the simulations, $k_{p f}=0.02 \mathrm{bar}^{-1}$ and $\omega_{p f}=15 \mathrm{rad} / \mathrm{s}$ resulted in an unstable system. Different values were tested in the laboratory, and a plot of different filter configurations is shown in Figure 24. High gain and low bandwidth yielded a stable system, and $k_{p f}=0.04 \mathrm{bar}^{-1}$ and $\omega_{p f}=1 \mathrm{rad} / \mathrm{s}$ were selected. 


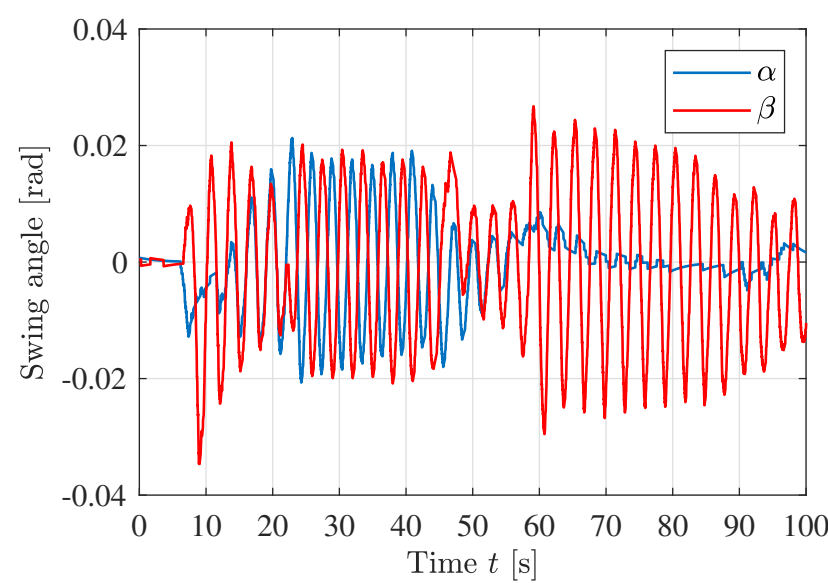

Figure 22: Swing angles without anti-swing control.

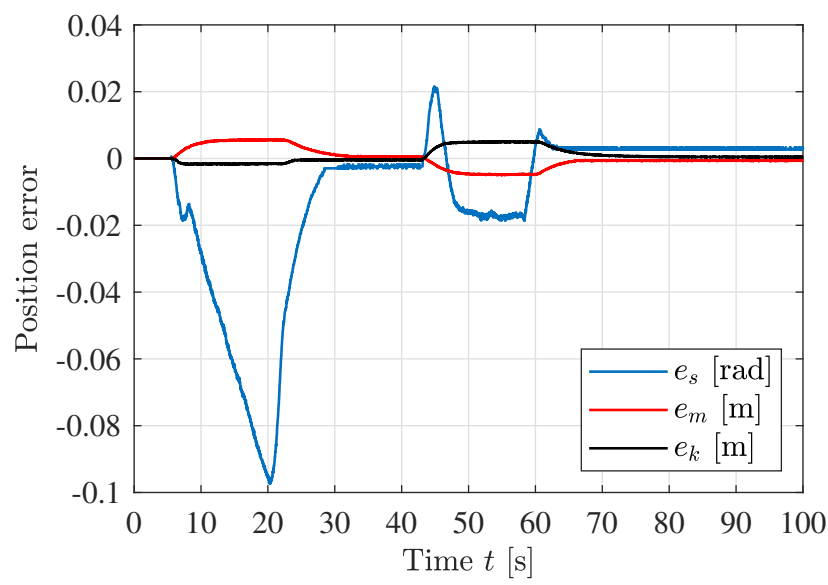

Figure 23: Position error without anti-swing control.

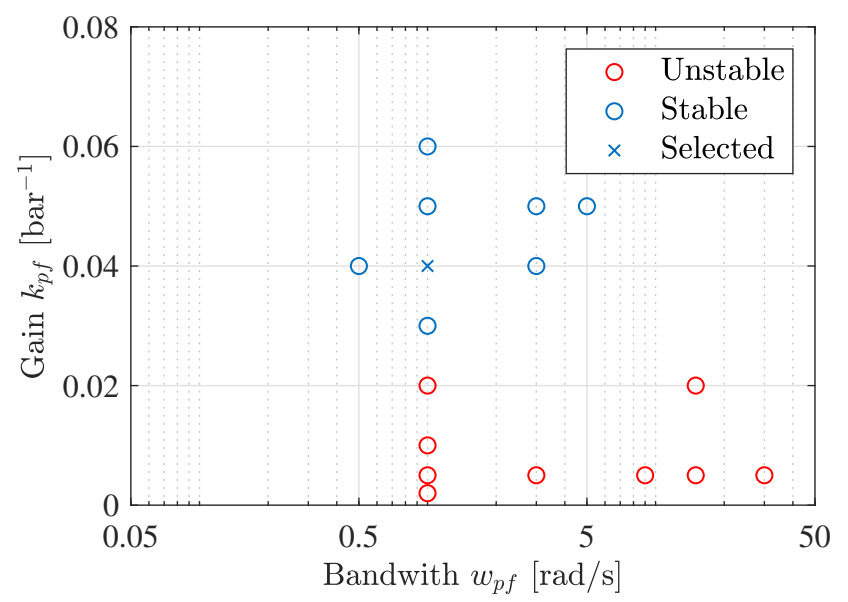

Figure 24: Stable and unstable pressure feedback configurations in the laboratory.
A plot of the swing angles without control is shown in Figure 25, showing good suppression of the oscillations, especially of $\alpha$. The angle $\beta$ still has some oscillations, which can be attributed to the fact that this angle is affected the most by the slewing motion and the instability issues mentioned. A plot of the position error with control is shown in Figure 26, again showcasing some difficulty to control the slewing motion. The control signal from the pressure feedback, before deadband compensation, is shown in Figure 27. It gives a large contribution, and even saturates at $t=60 \mathrm{~s}$.

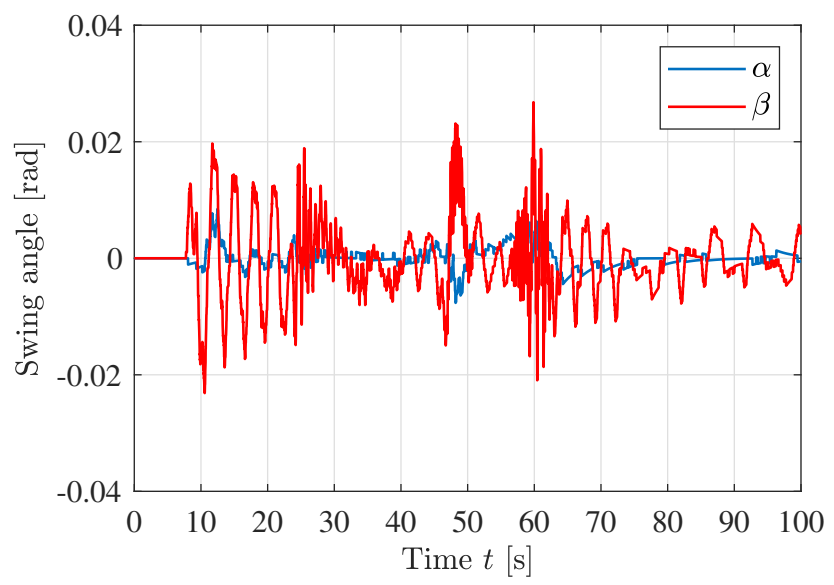

Figure 25: Swing angles with anti-swing control.

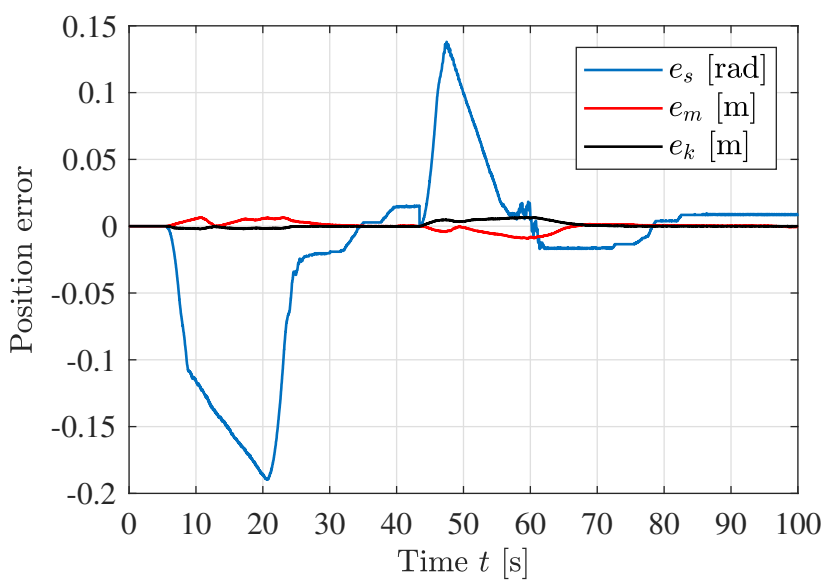

Figure 26: Position error with anti-swing control. 


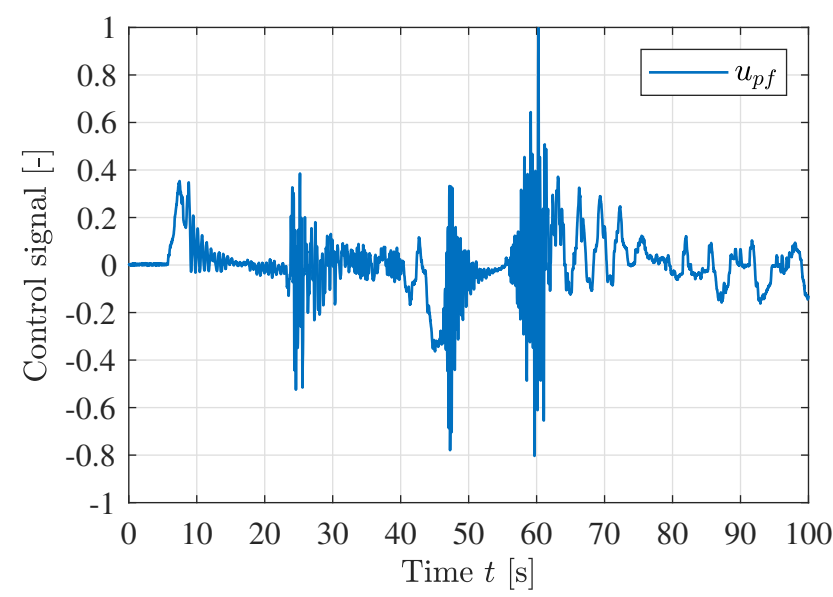

Figure 27: Control signal from pressure feedback.

The RMS values of the swing angles and position errors with and without anti-swing control is shown in Table 8 . The angle $\alpha$ is reduced by $75 \%$, with a slight impact on position error on the main and knuckle cylinder. The angle $\beta$ is reduced by $51 \%$, but has a large impact on the slew position error, quantifying the challenges faced with the slewing motion.

Table 8: Quantitative laboratory results.

\begin{tabular}{lll}
\hline Variable & No control & Anti-swing \\
\hline $\operatorname{RMS}(\alpha)[\mathrm{mrad}]$ & 7.19 & 1.75 \\
$\operatorname{RMS}(\beta)[\mathrm{mrad}]$ & 12.94 & 6.29 \\
$\operatorname{RMS}\left(e_{s}\right)[\mathrm{mrad}]$ & 26.12 & 66.88 \\
$\operatorname{RMS}\left(e_{m}\right)[\mathrm{mm}]$ & 2.91 & 3.26 \\
$\operatorname{RMS}\left(e_{k}\right)[\mathrm{mm}]$ & 2.19 & 2.36 \\
\hline
\end{tabular}

\section{Conclusion}

In this paper a 3D anti-swing controller for a hydraulic loader crane is designed, simulated, evaluated, and experimentally verified on a hydraulic loader crane. Relevant kinematic functions are derived to enable control of the swing angles. The motion control system operates in actuator space, and controls the three hydraulic cylinders in order to suppress the swing angles during motion. The kinematic functions are used to transform the feedback of the swing angles $\alpha$ and $\beta$ into command signals for the valves.

Simulation results show a large reduction in the swing angles using the anti-swing controller. Additionally, oscillations in the slewing motion were identified and suppressed by the introduction of pressure feedback. Quantitative analysis shows a slight increase in position error, but a $90 \%$ decrease in swing angles with the proposed controller. The increase in position error is expected as the suppression of the swing angles takes a higher priority.

Laboratory experiments show stability issues with the slewing motion when using anti-swing control, and pressure feedback was required to reach stability. Results show a large reduction of $75 \%$ for the in-plane angle $\alpha$, with a small impact on position error for the main and knuckle cylinders. The slewing motion proved more difficult to control, with a larger impact on the slew position error, but still a $51 \%$ reduction for the out-of-plane angle $\beta$ was obtained.

\section{Acknowledgments}

This work was supported by the Norwegian Ministry of Education and Research grant number 155597. 


\section{Appendix A. Solving the Euler-Lagrange Equations for Hanging Load Dynamics}

Selecting the coordinate $q=[\alpha \beta]^{T}$, the Euler-Lagrange equation is recalled as defined in Equation 6 .

$$
\frac{d}{d t} \frac{\partial \mathcal{L}}{\partial \dot{q}}-\frac{\partial \mathcal{L}}{\partial q}=0
$$

Expanding the Lagrangian $\mathcal{L}=\mathcal{K}-\mathcal{P}$ yields:

$$
\begin{aligned}
\mathcal{L}= & \frac{1}{2} m_{p}\left(\dot{x}_{t}^{2}+\dot{y}_{t}^{2}+\dot{z}_{t}^{2}+\dot{\alpha}^{2} L_{w}^{2}+c_{\alpha}^{2} \dot{\beta}^{2} L_{w}^{2}+\dot{L}_{w}^{2}+2 c_{\alpha} \dot{x}_{t} \dot{\alpha} L_{w}+2 s_{\alpha} \dot{x}_{t} \dot{L}_{w}-2 s_{\alpha} s_{\beta} \dot{y}_{t} \dot{\alpha} L_{w}\right. \\
& \left.+2 c_{\alpha} c_{\beta} \dot{y}_{t} \dot{\beta} L_{w}+2 c_{\alpha} s_{\beta} \dot{y}_{t} \dot{L}_{w}+2 s_{\alpha} c_{\beta} \dot{z}_{t} \dot{\alpha} L_{w}+2 c_{\alpha} s_{\beta} \dot{z}_{t} \dot{\beta} L_{w}-2 c_{\alpha} c_{\beta} \dot{z}_{t} \dot{L}_{w}\right)-m_{p} g\left(z_{t}-c_{\alpha} c_{\beta} L_{w}\right)
\end{aligned}
$$

Solving the Euler-Lagrange equation using the coordinate $\alpha$ yields:

$$
\begin{gathered}
\frac{\partial \mathcal{L}}{\partial \alpha}=m_{p}\left(-s_{\alpha} c_{\alpha} \dot{\beta}^{2} L_{w}^{2}-s_{\alpha} \dot{x}_{t} \dot{\alpha} L_{w}+c_{\alpha} \dot{x}_{t} \dot{L}_{w}-c_{\alpha} s_{\beta} \dot{y}_{t} \dot{\alpha} L_{w}-s_{\alpha} c_{\beta} \dot{y}_{t} \dot{\beta} L_{w}-s_{\alpha} s_{\beta} \dot{y}_{t} \dot{L}_{w}+c_{\alpha} c_{\beta} \dot{z}_{t} \dot{\alpha} L_{w}\right. \\
\left.-s_{\alpha} s_{\beta} \dot{z}_{t} \dot{\beta} L_{w}+s_{\alpha} c_{\beta} \dot{z}_{t} \dot{L}_{w}-g s_{\alpha} c_{\beta} L_{w}\right) \\
\frac{\partial \mathcal{L}}{\partial \dot{\alpha}}=m_{p}\left(\dot{\alpha} L_{w}^{2}+c_{\alpha} \dot{x}_{t} L_{w}-s_{\alpha} s_{\beta} \dot{y}_{t} L_{w}+s_{\alpha} c_{\beta} \dot{z}_{t} L_{w}\right) \\
\frac{d}{d t} \frac{\partial \mathcal{L}}{\partial \dot{\alpha}}=m_{p}\left(\ddot{\alpha} L_{w}^{2}+2 \dot{\alpha} L_{w} \dot{L}_{w}-s_{\alpha} \dot{x}_{t} \dot{\alpha} L_{w}+c_{\alpha} \ddot{x}_{t} L_{w}+c_{\alpha} \dot{x}_{t} \dot{L}_{w}\right. \\
-c_{\alpha} s_{\beta} \dot{y}_{t} \dot{\alpha} L_{w}-s_{\alpha} c_{\beta} \dot{y}_{t} \dot{\beta} L_{w}-s_{\alpha} s_{\beta} \ddot{y}_{t} L_{w}-s_{\alpha} s_{\beta} \dot{y}_{t} \dot{L}_{w} \\
\left.+c_{\alpha} c_{\beta} \dot{z}_{t} \dot{\alpha} L_{w}-s_{\alpha} s_{\beta} \dot{z}_{t} \dot{\beta} L_{w}+s_{\alpha} c_{\beta} \ddot{z}_{t} L_{w}+s_{\alpha} c_{\beta} \dot{z}_{t} \dot{L}_{w}\right) \\
\frac{d}{d t} \frac{\partial \mathcal{L}}{\partial \dot{\alpha}}-\frac{\partial \mathcal{L}}{\partial \alpha}=m_{p}\left(c_{\alpha} \ddot{x}_{t} L_{w}-s_{\alpha} s_{\beta} \ddot{y}_{t} L_{w}+s_{\alpha} c_{\beta} \ddot{z}_{t} L_{w}+\ddot{\alpha} L_{w}^{2}+2 \dot{\alpha}_{w} \dot{L}_{w}+s_{\alpha} c_{\alpha} \dot{\beta}^{2} L_{w}^{2}+g s_{\alpha} c_{\beta} L_{w}\right)=0
\end{gathered}
$$

Solving for $\ddot{\alpha}$ yields:

$$
\ddot{\alpha}=\frac{1}{L_{w}}\left(-c_{\alpha} \ddot{x}_{t}+s_{\alpha} s_{\beta} \ddot{y}_{t}-s_{\alpha} c_{\beta} \ddot{z}_{t}-2 \dot{\alpha} \dot{L}_{w}-s_{\alpha} c_{\alpha} \dot{\beta}^{2} L_{w}-g s_{\alpha} c_{\beta}\right)
$$

Solving the Euler-Lagrange equation using the coordinate $\beta$ yields:

$$
\begin{gathered}
\frac{\partial \mathcal{L}}{\partial \beta}=m_{p}\left(-s_{\alpha} c_{\beta} \dot{y}_{t} \dot{\alpha} L_{w}-c_{\alpha} s_{\beta} \dot{y_{t}} \dot{\beta} L_{w}+c_{\alpha} c_{\beta} \dot{y}_{t} \dot{L}_{w}-s_{\alpha} s_{\beta} \dot{z}_{t} \dot{\alpha} L_{w}+c_{\alpha} c_{\beta} \dot{z}_{t} \dot{\beta} L_{w}+c_{\alpha} s_{\beta} \dot{z}_{t} \dot{L}_{w}-g c_{\alpha} s_{\beta} L_{w}\right) \\
\frac{\partial \mathcal{L}}{\partial \dot{\beta}}=m_{p}\left(c_{\alpha}^{2} \dot{\beta} L_{w}^{2}+c_{\alpha} c_{\beta} \dot{y}_{t} L_{w}+c_{\alpha} s_{\beta} \dot{z}_{t} L_{w}\right) \\
\frac{d}{d t} \frac{\partial \mathcal{L}}{\partial \dot{\beta}}=m_{p}\left(-2 s_{\alpha} c_{\alpha} \dot{\alpha} \dot{\beta} L_{w}^{2}+c_{\alpha}^{2} \ddot{\beta} L_{w}^{2}+2 \dot{\beta} L_{w} \dot{L}_{w}\right. \\
-s_{\alpha} c_{\beta} \dot{y}_{t} \dot{\alpha} L_{w}-c_{\alpha} s_{\beta} \dot{y}_{t} \dot{\beta} L_{w}+c_{\alpha} c_{\beta} \ddot{y}_{t} L_{w}+c_{\alpha} c_{\beta} \dot{y}_{t} \dot{L}_{w} \\
\left.-s_{\alpha} s_{\beta} \dot{z}_{t} \dot{\alpha} L_{w}+c_{\alpha} c_{\beta} \dot{z}_{t} \dot{\beta} L_{w}+c_{\alpha} s_{\beta} \ddot{z}_{t} L_{w}+c_{\alpha} s_{\beta} \dot{z}_{t} \dot{L}_{w}\right)
\end{gathered}
$$




$$
\frac{d}{d t} \frac{\partial \mathcal{L}}{\partial \dot{\beta}}-\frac{\partial \mathcal{L}}{\partial \beta}=m_{p}\left(c_{\alpha} c_{\beta} \ddot{y}_{t} L_{w}+c_{\alpha} s_{\beta} \ddot{z}_{t} L_{w}-2 s_{\alpha} c_{\alpha} \dot{\alpha} \dot{\beta} L_{w}^{2}+2 \dot{\beta} L_{w} \dot{L}_{w}+c_{\alpha}^{2} \ddot{\beta} L_{w}^{2}+g c_{\alpha} s_{\beta} L_{w}\right)=0
$$

Solving for $\ddot{\beta}$ yields:

$$
\ddot{\beta}=\frac{1}{c_{\alpha} L_{w}}\left(-c_{\beta} \ddot{y}_{t}-s_{\beta} \ddot{z}_{t}+2 s_{\alpha} \dot{\alpha} \dot{\beta} L_{w}-2 \dot{\beta} \dot{L}_{w}-g s_{\beta}\right)
$$

\section{References}

Ambrosino, M., Dawans, A., Thierens, B., and Garone, E. Oscillation reduction for knuckle cranes. In Proceedings of the 37th International Symposium on Automation and Robotics in Construction (ISARC). International Association for Automation and Robotics in Construction (IAARC), Kitakyushu, Japan, pages 1590-1597, 2020. doi:10.22260/ISARC2020/0221.

Bak, M. K. and Hansen, M. R. Analysis of offshore knuckle boom crane part two: Motion control. Modeling, Identification and Control, 2013. 34(4):175181. doi:10.4173/mic.2013.4.2.

Boschetti, G., Caracciolo, R., Richiedei, D., and Trevisani, A. A non-time based controller for load swing damping and path-tracking in robotic cranes. Journal of Intelligent \& Robotic Systems, 2014. 76(2):201-217. doi:10.1007/s10846-014-0036-7.

Boschetti, G., Richiedei, D., and Trevisani, A. Delayed reference control for multi-degree-of-freedom elastic systems: Theory and experimentation. Control Engineering Practice, 2011. 19(9):1044 - 1055. doi:10.1016/j.conengprac.2011.05.006.

Cho, S.-K. and Lee, H.-H. A fuzzy-logic antiswing controller for three-dimensional overhead cranes. ISA Transactions, 2002. 41(2):235 - 243. doi:10.1016/S0019-0578(07)60083-4.

Fang, Y., Dixon, W. E., Dawson, D. M., and Zergeroglu, E. Nonlinear coupling control laws for an underactuated overhead crane system. IEEE/ASME Transactions on Mechatronics, 2003. 8(3):418-423. doi:10.1109/TMECH.2003.816822.

Hagen, D., Padovani, D., and Choux, M. Design and implementation of pressure feedback for loadcarrying applications with position control. In Proceedings of the Sixteenth Scandinavian International Conference on Fluid Power. Tampere, Finland, 2019.

Ho-Hoon Lee and Seung-Gap Choi. A model-based anti-swing control of overhead cranes with high hoisting speeds. In Proceedings 2001 ICRA. IEEE
International Conference on Robotics and Automation (Cat. No.01CH37164), volume 3. pages 25472552 vol.3, 2001. doi:10.1109/ROBOT.2001.933006.

Ho-Hoon Lee and Sung-Kun Cho. A new fuzzy-logic anti-swing control for industrial three-dimensional overhead cranes. In Proceedings 2001 ICRA. IEEE International Conference on Robotics and Automation (Cat. No.01CH37164), volume 3. pages 29562961 vol.3, 2001. doi:10.1109/ROBOT.2001.933070.

Jensen, K. J., Ebbesen, M. K., and Hansen, M. R. Adaptive feedforward control of a pressure compensated differential cylinder. Applied Sciences, 2020a. 10(21):7847. doi:10.3390/app10217847.

Jensen, K. J., Ebbesen, M. K., and Hansen, M. R. Anti-swing control of a hydraulic loader crane with a hanging load. Mechatronics, 2021. 77:102599. doi:10.1016/j.mechatronics.2021.102599.

Jensen, K. J., Kjeld Ebbesen, M., and Rygaard Hansen, M. Development of point-topoint path control in actuator space for hydraulic knuckle boom crane. Actuators, 2020b. 9(2):27. doi:10.3390/act9020027.

Kjelland, M. B. and Hansen, M. R. Using input shaping and pressure feedback to suppress oscillations in slewing motion of lightweight flexible hydraulic crane. International Journal of Fluid Power, 2015. 16(3):141-148. doi:10.1080/14399776.2015.1089071.

Kjelland, M. B., Hansen, M. R., Tyapin, I., and Hovland, G. Tool-point control of a planar hydraulically actuated manipulator with compensation of non-actuated degree of freedom. In 2012 12th International Conference on Control, Automation and Systems. pages 672-677, 2012.

Kjelland, M. B., Tyapin, I., Hovland, G., and Hansen, M. R. Tool-point control for a redundant heave compensated hydraulic manipulator. Proceedings of the 2012 IFAC Workshop on Automatic Control in Offshore Oil and Gas Production, Norwegian University of Science and Technology, Trondheim, Norway, 2012. doi:10.3182/20120531-2-NO-4020.00034. 
Lee, H.-H. Modeling and Control of a ThreeDimensional Overhead Crane. Journal of Dynamic Systems, Measurement, and Control, 1998. 120(4):471-476. doi:10.1115/1.2801488.

Lee, H.-H. A new approach for the anti-swing control of overhead cranes with high-speed load hoisting. International Journal of Control, 2003. 76(15):14931499. doi:10.1080/00207170310001604954.

Lee, H.-H. A new design approach for the anti-swing trajectory control of overhead cranes with high-speed hoisting. International Journal of Control, 2004 . 77(10):931-940. doi:10.1080/00207170412331270550.

Lee, H.-H., Cho, S.-K., and Cho, J.-S. A new antiswing control of overhead cranes. IFAC Proceedings Volumes, 1997. 30(13):115 - 120. doi:10.1016/S14746670(17)44380-1. IFAC Workshop on Automation in the Steel Industry: Current Practice and Future Developments (ASI'97), Kyongju, Korea, 16-18 July 1997.

Lee, H.-H. and Liang, Y. A Robust Anti-Swing Trajectory Control of Overhead Cranes With High-Speed Load Hoisting: Experimental Study. 2010. Volume 8: Dynamic Systems and Control, Parts A and B:711-716. doi:10.1115/IMECE2010-39708.

Lee, H.-H., Liang, Y., and Segura, D. A Sliding-Mode Antiswing Trajectory Control for Overhead Cranes With High-Speed Load Hoisting. Journal of Dynamic Systems, Measurement, and Control, 2006. 128(4):842-845. doi:10.1115/1.2364010.

Ngo, Q. H. and Hong, K. Sliding-mode antisway control of an offshore container crane. IEEE/ASME Transactions on Mechatronics, 2012. 17(2):201-209. doi:10.1109/TMECH.2010.2093907.

Park, H., Chwa, D., and Hong, K.-S. A feedback linearization control of container cranes: Varying rope length. International Journal of Control, Automation, and Systems, 2007. 5.
Park, M., Chwa, D., and Hong, S. Antisway tracking control of overhead cranes with system uncertainty and actuator nonlinearity using an adaptive fuzzy sliding-mode control. IEEE Transactions on Industrial Electronics, 2008. 55(11):3972-3984. doi:10.1109/TIE.2008.2004385.

Pedersen, H. C. and Andersen, T. O. Pressure feedback in fluid power systemsactive damping explained and exemplified. IEEE Transactions on Control Systems Technology, 2018. 26(1):102-113. doi:10.1109/TCST.2017.2650680.

Pedersen, H. C., Andersen, T. O., and Hansen, M. R. Guidelines for properly adjusting pressure feedback in systems with over-centre valves. In Proceedings of the BATH/ASME 2016 Symposium on Fluid Power and Motion Control. Bath, UK, 2016. doi:10.1115/FPMC2016-1780.

Schindele, D., Menn, I., and Aschemann, H. Nonlinear optimal control of an overhead travelling crane. In 2009 IEEE Control Applications, (CCA) Intelligent Control, (ISIC). pages 1045-1050, 2009. doi:10.1109/CCA.2009.5280705.

Singhose, W., Seering, W., and Singer, M. Input shaping for vibration reduction with specified insensitivity to modeling errors. Proc. Japan-USA Symp. Flexible Automation, 1996. 1.

Sorensen, K. L., Singhose, W., and Dickerson, S. A controller enabling precise positioning and sway reduction in bridge and gantry cranes. Control Engineering Practice, 2007. 15(7):825 - 837. doi:10.1016/j.conengprac.2006.03.005. Special Issue on Award Winning Applications.

Sung-Kun Cho and Ho-Hoon Lee. An anti-swing control of a 3-dimensional overhead crane. In Proceedings of the 2000 American Control Conference. ACC (IEEE Cat. No.00CH36334), volume 2. pages 10371041 vol.2, 2000. doi:10.1109/ACC.2000.876658. 